\title{
Cross-Hemispheric Complementary Prefrontal Mechanisms during Task Switching under Perceptual Uncertainty
}

\author{
${ }^{\circledR}$ Kaho Tsumura, ${ }^{1}{ }^{\oplus}$ Ryuta Aoki, ${ }^{2}{ }^{\oplus}$ Masaki Takeda, ${ }^{2}{ }^{\circledR}$ Kiyoshi Nakahara, ${ }^{2}$ and ${ }^{\oplus}$ Koji Jimura ${ }^{1,2}$ \\ ${ }^{1}$ Department of Biosciences and Informatics, Keio University, Yokohama 223-0061, Japan, and ${ }^{2}$ Research Center for Brain Communication, Kochi \\ University of Technology, Kami 782-8502, Japan
}

Flexible adaptation to changing environments is a representative executive control function implicated in the frontoparietal network that requires appropriate extraction of goal-relevant information through perception of the external environment. It remains unclear, however, how the flexibility is achieved under situations where goal-relevant information is uncertain. To address this issue, the current study examined neural mechanisms for task switching in which task-relevant information involved perceptual uncertainty. Twenty-eight human participants of both sexes alternated behavioral tasks in which they judged motion direction or color of visually presented colored dot stimuli that moved randomly. Task switching was associated with frontoparietal regions in the left hemisphere, and perception of ambiguous stimuli involved contralateral homologous frontoparietal regions. On the other hand, in stimulus-modality-dependent occipitotemporal regions, task coding information was increased during task switching. Effective connectivity analysis revealed that the frontal regions signaled toward the modality-dependent occipitotemporal regions when a relevant stimulus was more ambiguous, whereas the occipitotemporal regions signaled toward the frontal regions when the stimulus was more distinctive. These results suggest that complementary prefrontal mechanisms in the left and right hemispheres help to achieve a behavioral goal when the external environment involves perceptual uncertainty.

Key words: behavioral flexibility; executive control; occipitotemporal; perceptual decision-making; prefrontal

Significance Statement

In our daily life, environmental information to achieve a goal is not always certain, but we make judgments in such situations, and change our behavior accordingly. This study examined how the flexibility of behavior is achieved in a situation where goal-relevant information involves perceptual uncertainty. fMRI revealed that the lateral prefrontal cortex (PFC) in the left hemisphere is associated with behavioral flexibility, and the perception of ambiguous stimuli involves the PFC in the right hemisphere. These bilateral PFC signaled to stimulus-modality-dependent occipitotemporal regions, depending on perceptual uncertainty and the task to be performed. These top-down signals supplement task coding in the occipitotemporal regions, and highlight interhemispheric prefrontal mechanisms involved in executive control and perceptual decision-making.

\section{Introduction}

Executive control guides flexible adaptation to changing environments, which has been most developed in humans through evolution (Roberts et al., 1998; Fuster, 2015), and is implemented in distributed frontoparietal cortical regions (Miller and Cohen,

\footnotetext{
Received Aug. 9, 2020; revised Jan. 5, 2021; accepted Jan. 7, 2021

Author contributions: K.T. and K.J. designed research; K.T., R.A., K.N., and K.J. performed research; K.T. and K.J. contributed unpublished reagents/analytic tools; K.T. and K.J. analyzed data; K.T., M.T., and K.J. wrote the first draft of the paper; K.T., R.A., M.T., K.N., and K.J. edited the paper; K.T., M.T., and K.J. wrote the paper.

This work was supported by Japan Society for the Promotion of Science Kakenhi 26350986, 26120711, 17H05957, 17K01989, and $20 \mathrm{~K} 07727$ to K.J.; 17H00891to K.N.; $20 \mathrm{H} 00521$ to M.T.; Uehara Memorial Foundation to K.J.; and Takeda Science Foundation to K.J. We thank Maoko Yamanaka for administrative assistance; and Tetsumi Tokita for technical assistance.

The authors declare no competing financial interests.

Correspondence should be addressed to Koji Jimura at jimura@bio.keio.ac.jp.

https://doi.org/10.1523/JNEUROSCI.2096-20.2021

Copyright $(2021$ the authors
}

2001; Dosenbach et al., 2006; Duncan, 2010). Shifting between different types of behaviors is a representative executive control function (Jersild, 1927; Allport et al., 1994; Rogers and Monsell, 1995; Monsell, 2003), and task switching paradigms have often been used to investigate neural mechanisms of behavioral flexibility. Previous neuropsychological and neuroimaging studies suggest that the posterior part of the left PFC (IPFC) plays a critical role in task switching (Fig. 1A) (Konishi et al., 1998, 2002; Dove et al., 2000; Rushworth et al., 2002; Crone et al., 2006; Brass and von Cramon, 2004; Derrfuss et al., 2005; Yeung et al., 2006; C. Kim et al., 2012).

On the other hand, goal-relevant sensory information from the external environment is perceived and guides a course of action, which is referred to as perceptual decision-making. Studies of perceptual decision-making have used behavioral tasks that demand discrimination of presented sensory stimuli involving perceptual uncertainty (Newsome and Pare, 1988; Corbetta 
A

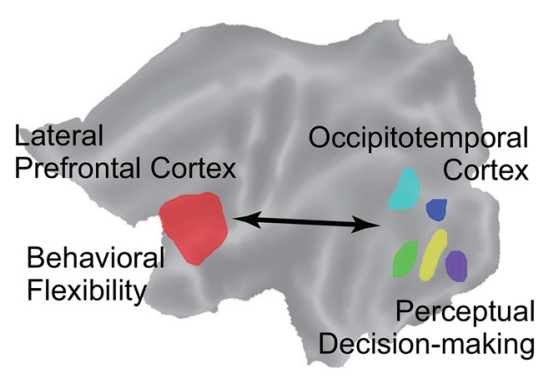

B

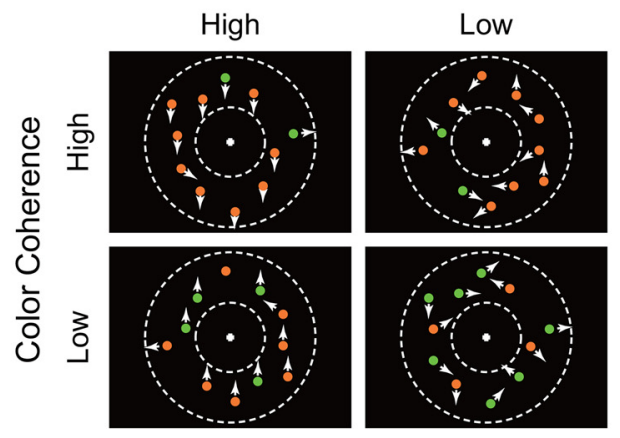

Stimulus

Motion Coherence
C
0.7
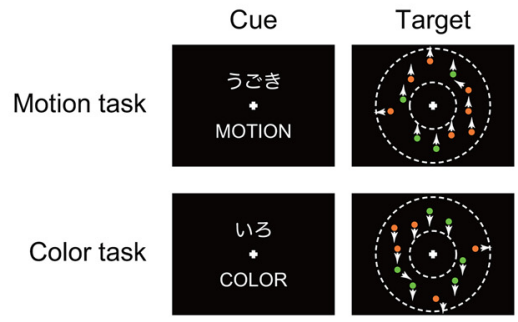

0.3

Coherence

Response

$80 \%, 40 \%, 20 \%$ Task sequence

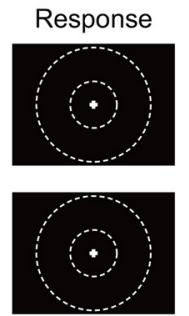

1.5

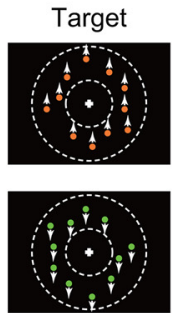

0.3

$100 \%$
D

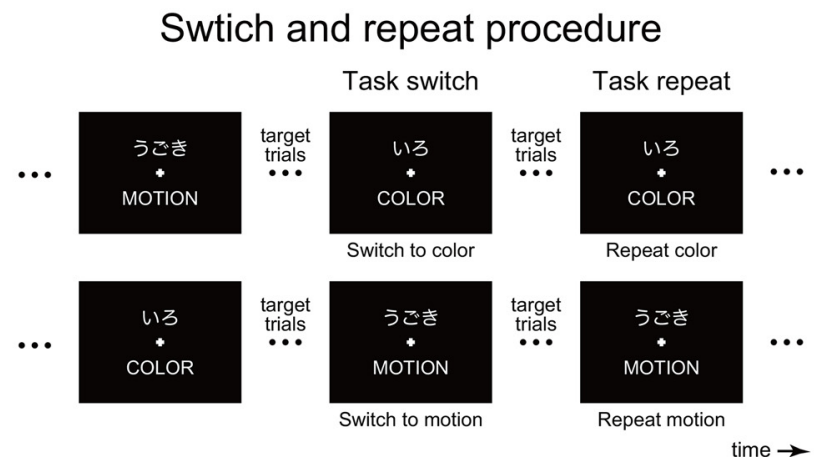

Figure 1. Study design. $\boldsymbol{A}$, Behavioral flexibility involves the engagement of the lateral PFC and requires perceptual decision-making implicated in the occipitotemporal regions where modality-dependent mechanisms are distributed. $\boldsymbol{B}$, Visual stimuli. Coherence of random dot motion and color were independently manipulated. White dots were presented within a donut-shaped display circle as indicated by the dotted lines. Arrow indicates the overall motion direction of each dot. C, Task sequence. After the presentation of a task cue (MOTION or COLOR), the visual stimulus was presented, and participants judged the overall motion or color of the stimuli and pressed a corresponding button. They continued the judgment along the same dimension until the next cue was presented. Only the trials immediately after the cue presentation involved perceptual uncertainty, and subsequent trials did not involve uncertainty (100\% coherent). $\boldsymbol{D}$, Task switch and task repeat. If the presented cue was different from the former one, the task to be performed was switched (left); otherwise, the task was repeated (right).

et al., 1991; Erwin et al., 1992; Romo et al., 1998). In one common task, randomly moving dots are presented visually, and participants are required to judge the overall direction of the movement or the overall color of the dot stimuli. It is known that the middle temporal (MT) region plays an important role in perceptual decision-making for moving stimuli (Britten et al., 1992; Shadlen et al., 1996; Beauchamp et al., 1997; Braddick et al., 2001; Corbetta and Shulman, 2002; Huk et al., 2002; Mazurek et al., 2003; Kayser et al., 2010). On the other hand, it has been suggested that ventral visual areas, including V4 and the ventral visual complex (VVC), are associated with the perception of color stimuli (Schein and Desimone, 1990; Motter, 1994; Bartels and
Zeki, 2000; Kayser et al., 2010). The MT and VVC/V4 involvements reflect perception of goal-relevant information rather than simple accumulation of stimulus evidence (Kayser et al., 2010). These results suggest stimulus-modality-dependent mechanisms distributed across occipitotemporal regions for the perception of visual information (Fig. $1 \mathrm{~A}$ ).

Executive control depends on the perceived information of the external environment, and the perceived information should be relevant to the achievement of behavioral goals. In other words, executive control presumes that relevant information is appropriately extracted by the perception of the external environment (Fig. 1A). In our daily life, however, goal-relevant 
environmental information is not always evident. Although most prior studies of executive control have used sensory stimuli that could be perceived distinctively (e.g., Krystal et al., 1994; Rogers and Monsell, 1995; Casey et al., 1997; MacDonald et al., 2000; Miyake et al., 2000; Braver et al., 2003; Aron et al., 2004; Seeley et al., 2007), several studies of perceptual discrimination used stimuli for which multidimensional visual uncertainties were manipulated within a stimulus set (Kayser et al., 2010; Mante et al., 2013; Zhang et al., 2013; Kumano et al., 2016). However, to our knowledge, task switching has been rarely explored in behavioral situations where environmental information involves perceptual uncertainties.

The current study aimed to elucidate relationships between task switching and perceptual decision-making, and to explore the underlying neural mechanisms. We hypothesized three independent mechanisms: (1) a functionally merged single region implements task switching and perceptual uncertainty (e.g., Shackman et al., 2011; Yarkoni et al., 2011); (2) distinct regions for perceptual decision-making and task switching interact to guide task switching (e.g., Konishi et al., 1998; Egner and Hirsch, 2005; Kayser et al., 2010; Waskom et al., 2014); and (3) a hublike region links the regions for task switching and perceptual decision-making (e.g., Cole et al., 2013; Osada et al., 2015; Nee and D’Esposito, 2016; Jiang et al., 2018).

To test these three hypotheses, fMRI was administered while human participants alternated tasks in which the motion strength and color strength of randomly moving dots were independently manipulated as perceptual uncertainties (Fig. 1B-D). We first explored the brain regions associated with task switching, motion strength, and color strength. Then whole-brain exploratory multivariate pattern analysis (MVPA) was performed to identify brain regions involving task-relevant information. Finally, to examine functional network mechanisms, effective connectivity was examined among the task-related brain regions during task switching under conditions of perceptual uncertainty.

\section{Materials and Methods \\ Participants}

Written informed consent was obtained from 28 healthy right-handed subjects (age range: 18-23 years; 10 females). The experimental procedures were approved by the institutional review boards of Keio University (Yokohama, Japan) and Kochi University of Technology (Kami, Japan). Participants received 2000 yen for participating in each session. One participant was excluded from the analysis because of low behavioral performance. All participants had normal color vision. The number of participants was determined before the collection of the current data based on the effect sizes in relevant previous and pilot behavioral experiments $\left(\alpha=0.05, \beta=0.95, \eta^{2}=0.42\right)$.

\section{Experimental design}

Behavioral procedures. The experiment consisted of two sessions administered on separate days. The first day was training sessions, in which participants practiced two discrimination tasks (color and motion) and switching between these two tasks. The second day was scanning sessions, and the participants performed the switching task using the motion dot stimuli identical to those used in the training sessions.

Stimuli. All stimuli were generated in MATLAB version 2012a, using the Psychophysics Toolbox extension version 3.0.10 (Brainard, 1997; Pelli, 1997), and were presented visually on a computer screen. The current stimuli were similar to those used in a previous study of perceptual decision-making (Chen et al., 2015) (Fig. 1B), but each dot was colored in red or green. Each motion stimulus involved 75 dots moving inside a donut-shaped display patch with a white cross in the center of the patch on a black background (Fig. 1B). The display patch and cross were centered on the screen and extended from 6 to 12 degrees of visual angle. Within the display patch, every dot moved at a speed of 10 degrees of visual angle per second. Some dots moved coherently in one direction (up or down), whereas the others moved randomly. The percentage of coherently moving dots determined the "motion coherence," and three levels $(20 \%, 40 \%$, and $80 \%)$ of motion coherence were used.

Dot presentation was controlled to remove local motion signals following a standard method for generating motion stimuli (Newsome and Pare, 1988; Britten et al., 1993; Shadlen and Newsome, 1994; Palmer et al., 2005). Namely, on stimulus onset, the dots were presented at new random locations on each of the first three frames. They were relocated after two subsequent frames, such that the dots in frame 1 were repositioned in frame 4 , the dots in frame 2 were repositioned in frame 5, etc. When repositioned, each dot was either randomly presented at the new location or aligned with the predetermined motion direction, depending on the predetermined motion strength for that trial. Each stimulus was composed of 18 video frames with $60 \mathrm{~Hz}$ refresh rates (i.e., $300 \mathrm{~ms}$ presentation).

Each dot was either orange $(\mathrm{RGB}=[255,123,11]$; HSV $=[27,95$, $100])$ or green $(\operatorname{RGB}=[0,255,0] ; \operatorname{HSV}=[120,100,100])$, and the two colors were equally bright $(\mathrm{V}=100)$. The percentage of red dots in the dot stimuli was defined as "color coherence," and three levels (20\%, 40\%, and $80 \%$ ) of color coherence were used. Then, the color coherence and motion coherence were independently manipulated for the dot stimuli.

Task procedure. At the beginning of the task, a task cue (MOTION or COLOR) was presented, indicating the dimension of discrimination in subsequent trials (Fig. 1C). Then, the dot patch was presented. In the motion task, participants were required to judge the direction of overall motion of the patch (up or down); in the color task, they were required to judge the overall color (orange or green). They then pressed a corresponding button (right or left) with their right thumb as quickly and accurately as possible. If participants made an incorrect response, or did not respond within $1.8 \mathrm{~s}$ from the onset of the dot stimuli, a feedback stimulus indicating the error $(\mathrm{X})$ was presented for $1.0 \mathrm{~s}$, followed by a cue presentation for the same task. The stimulus-response association for the two tasks was identical on days 1 and 2, and was counterbalanced across participants.

For the task switching design, we applied an intermittent cueing paradigm that requires participants to actively maintain a task set until the next cue presentation, which enhances the switching effect (Sakai, 2008). Specifically, after a cue was presented, the dot target trial was repeated 46 times, and the participants identified the dot stimulus along the same dimension until the next cue stimulus was presented. Motion and color coherence levels of the dot patch in these trials (second to sixth trials after cue presentation) were set to $100 \%$ and did not involve uncertainty, which assured successive correct trials until the next task cue was presented to enhance the task switch effect (see also Behavioral analysis). After the participants gave successive correct responses in 4-6 trials, the next task cue was presented.

One task block lasted $\sim 90 \mathrm{~s}$, which involved 5-7 task-cue presentations. Fixation blocks were also presented for $20 \mathrm{~s}$ between task blocks. One functional run consisted of three task blocks and two fixation blocks. Each run lasted for $305 \mathrm{~s}$.

Practice procedure. On the first day, participants practiced behavioral tasks outside the scanner. They first practiced stimulus discrimination tasks for dot stimuli, where only one task dimension (color or motion) was repeatedly presented (i.e., single-task run). Participants alternatively practiced a motion or color discrimination task for every 1 or 2 practice runs, and completed a total of 8 practice single-task runs.

They then practiced switching tasks, in which a task cue was pseudorandomly alternated (i.e., task-switching run; Fig. 1D). During taskswitching runs, the dot target trial that took place immediately after the cue presentation involved perceptual uncertainty manipulated by dot color and motion coherence, as in practice runs; however, after the first target trials until the next cue presentation, the dot target involved no uncertainty (i.e., $100 \%$ coherence for both color and motion; Fig. $1 C$; see also above). Participants performed two practice runs for the taskswitching runs. 
Behavioral procedure in scanning session. On the second day, after practicing a taskswitching run, they performed nine taskswitching runs using the same procedure as that was used on day 1 while fMRI was administered.

Imaging procedure. MRI scanning was administered using a 3T MRI scanner (Siemens Verio) with a 32-channel head coil. Functional images were acquired using a multiband acceleration EPI sequence (TR: $0.8 \mathrm{~s}$; TE: $30 \mathrm{~ms}$; flip angle: $45 \mathrm{deg}$; 80 slices; slice thickness: $2 \mathrm{~mm}$; in-plane resolution: $3 \times 3 \mathrm{~mm}$; multiband factor: 8) (Moeller et al., 2010). Each functional run involved 385 volume acquisitions. The first 10 volumes were discarded for analysis to take into account the equilibrium of longitudinal magnetization. High-resolution anatomic images were acquired using an MP-RAGE T1-weighted sequence (TR: $2500 \mathrm{~ms}$; TE $=4.32 \mathrm{~ms}$; flip angle: 8 deg; 192 slices; slice thickness: $1 \mathrm{~mm}$; in-plane resolution: $\left.0.9 \times 0.9 \mathrm{~mm}^{2}\right]$ ).

\section{Statistical analysis}

Behavioral analysis. Two types of target trials were of interest: (1) task-switching trials immediately after cue presentation indicating a task alternation (i.e., switch to the color task from the motion task or vice versa); and (2) task-repeat trials immediately after cue presentation, indicating a repeat of the previous task. These trials were further classified in terms of the performed task (motion and color), and the coherence level of the dot stimulus (20\%, $40 \%$, and $80 \%$ ), entailing a $2 \times 2$ $\times 3$ factorial model (switch, task, coherence). The trials that occurred after an error were excluded from the entire analysis. Trials with $100 \%$ coherence after the task switching and repeat trials were classified as either motion or color trials. Then, accuracy and reaction time were calculated for each condition. Statistical tests were performed based on repeatedmeasures ANOVAs implemented using SPSS Statistics 24 (IBM).

Image preprocessing. MRI data were analyzed using SPM12 software (https://www.fil.ion.ucl.ac.uk/spm/). All functional images were first temporally realigned across volumes and runs, and the anatomic image was coregistered to a mean image of the functional images. The functional images were then spatially normalized to a standard MNI template with normalization parameters estimated for the anatomic scans. The images were resampled into $2 \mathrm{~mm}$ isotropic voxels, and spatially smoothed with a $6 \mathrm{~mm}$ FWHM Gaussian kernel.

Imaging analysis: General linear model First level analysis. A GLM approach (Worsley and Friston, 1995) was used to estimate parameter values for task events. The events of interest were correct switch, repeat, and $100 \%$ coherent trials, and were coded for motion and color tasks separately. For switch and repeat trials, the normalized (demeaned and divided by SD) coherence level of relevant stimuli (motion coherence for motion task or color coherence for color task) was added as a parametrical effect of interest. Error trials were coded in GLM as nuisance effects. Those task events were time-locked to the onset of random dot stimuli and then convolved with a canonical HRF implemented in SPM. Additionally, six axis head movement parameters, white matter signal, CSF signal, and the parametrical effect of reaction times were included in GLM as nuisance effects. Then, parameters were estimated for each voxel across the whole brain.

Group-level analysis. Maps of parameter estimates were first contrasted within individual participants. The contrasted maps were collected from participants and were subjected to group-mean tests. Voxel clusters were first identified using a voxelwise uncorrected threshold of $p<0.001$. The voxel clusters were then tested for a significance across the whole brain with a threshold of $p<0.05$ corrected by family-wise error rate based on permutation methods (5000 permutations) implemented in randomize in FSL suite (www.fmrib.ox.ac.uk/fsl/), which was
B

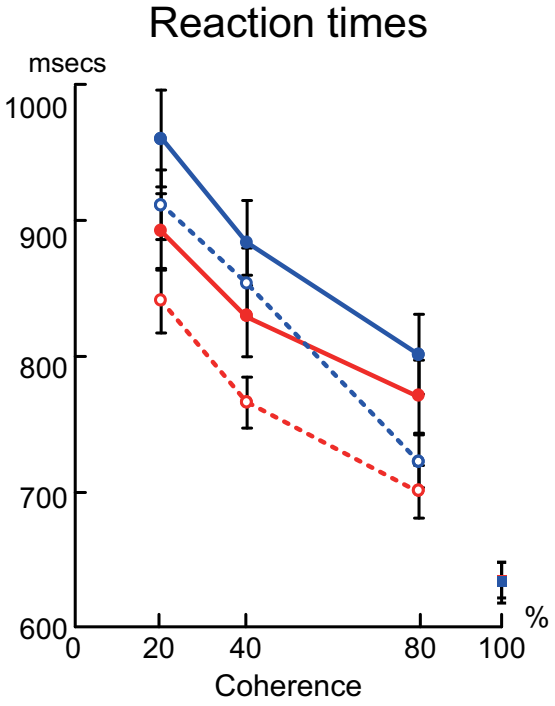

empirically validated to appropriately control the false positive rate in a previous study (Eklund et al., 2016). To identify occipitotemporal cortical regions specifically associated with the color task (color minus motion tasks), a mask obtained from Neurosynth (http://neurosynth.org/) for the search word "color" ( $z>3.0$, for uniformity test) was used. Peaks of significant clusters were then identified and listed in tables. If multiple peaks were identified within $12 \mathrm{~mm}$, the most significant peak was kept.

Exploration of hemispheric laterality. To examine the hemispheric laterality of the task effects, we explored brain regions showing greater task effects than the contralateral homologous regions. For each participant, contrast maps were flipped along the $x$ (left-right) axis and were subtracted from the original nonflipped maps on a voxel-by-voxel basis (Konishi et al., 2002). Then, the group-level statistical significance was tested within the right hemisphere. Statistical correction was performed within one hemisphere because the subtracted maps show sign-flipped symmetry along the $x$ axis.

We applied this procedure to the maps of the coherence effect (motion and color collapsed) and the switch effect (switch vs repeat). For the coherence effect, the maps were sign-flipped and had a greater signal in the low-coherent trial. Thus, positive values of hemispheric laterality indicate a greater signal in low-coherent trials in the right hemisphere and/or a lower signal in high coherent trials in the left hemisphere. Likewise, for the switch effect, positive values indicate greater activation during switch versus repeat trials in the right hemisphere and/or lower activation switch versus repeat trials in the left hemisphere. The negative values indicate the reverse effects.

ROI analysis for hemispheric laterality. The exploratory analysis above assumes a homology of the brain regions in the right and left hemispheres. We next performed an ROI analysis without assuming hemispheric homology. ROIs were defined as brain regions identified as a multiple demand network (Camilleri et al., 2018). Specifically, ROIs were classified as being in the left or right regions based on the $x$ axis coordinates (Camilleri et al., 2018, their Table 2). ROI images were created with spheres that had a radius of $6 \mathrm{~mm}$ and were centered at the coordinates. Then, for each of the left and right ROIs, the estimation contrast of switch effect (switch vs repeat) and the parametric effect of the coherence were extracted from each participant. Group-level statistics were calculated treating participants as a random effect.

To test the robustness of the ROI analysis against the ROI definition, we redefined ROIs. Image maps of meta-analyses were obtained from 


\section{A}

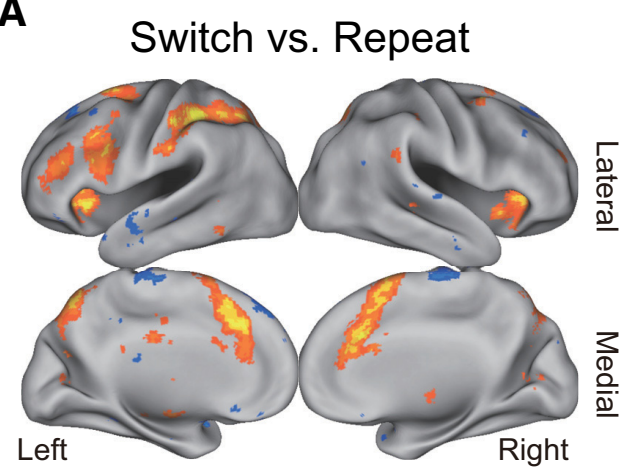

C

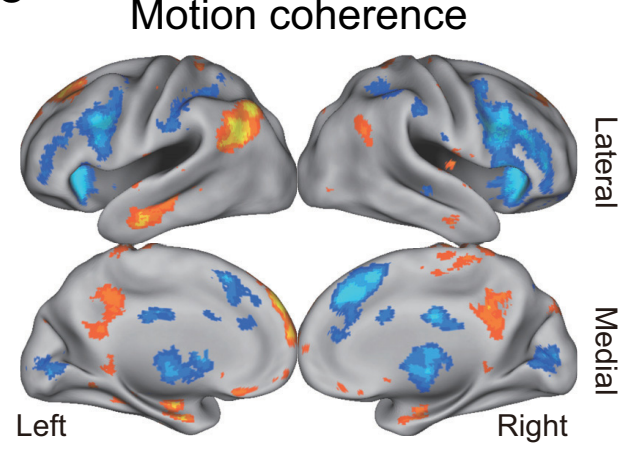

B

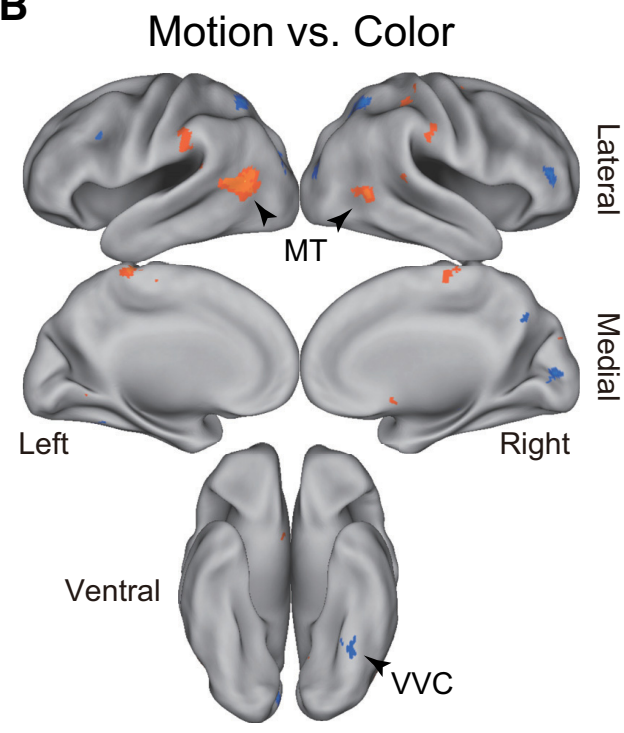

D

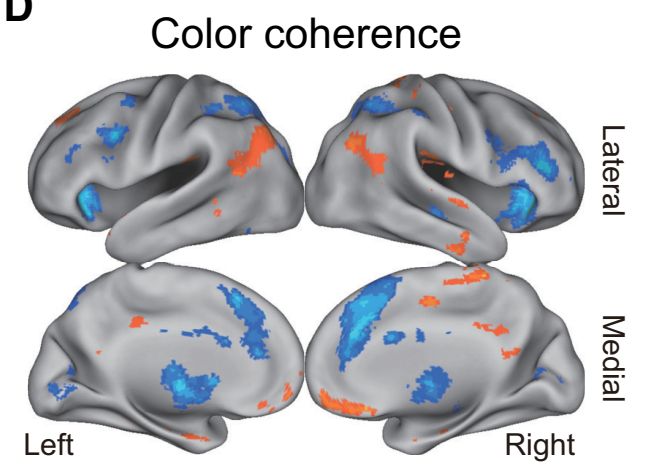

$$
z=3.1 \quad z=5.6
$$$$
z=-3.1 \quad z=-5.6
$$

Figure 3. Statistical activation map of univariate analysis. Maps are overlaid onto 3D surface of the brain. Hot colors represent positive effects. Cool colors represent negative effects. $\boldsymbol{A}$, Switch versus repeat trials. $\boldsymbol{B}$, Motion versus color tasks. $\boldsymbol{C}$, Motion coherence effect. Hot colors represent a greater signal in high-coherence trials. Cool colors represent a greater signal in lowcoherence trials. $\boldsymbol{D}$, Color coherence effect. The format is similar to those in $\boldsymbol{C}$.

Neurosynth (Yarkoni et al., 2011) using the terms "switching" (https:// neurosynth.org/analyses/terms/switching/) and "task demands" (https:// neurosynth.org/analyses/terms/task\%20demands/). A threshold of $p<$ 0.01 (uniformity test with whole-brain correction based on false discovery rate) was set for each map. Then, an image map of the logical sum of the two maps with thresholds was created, and ROIs were defined as being in the left or right hemisphere based on the $x$ axis coordinates. Regions with $X=0$ were excluded from the analysis. The same statistical tests were performed using these ROIs.

MVPA. To explore brain regions representing task-related information, an MVPA was performed. Bivariate classification based on a support vector machine was used to decode a performed task (motion or color). A searchlight procedure (Kriegeskorte et al., 2006) with a 5 voxel radius was used to provide a measure of decoding accuracy in the neighborhood of each voxel. Training and testing were performed based on the Decoding Toolbox (TDT; version 3.95; https://sites.google.com/site/ tdtdecodingtoolbox/).

For each functional run, a single-level GLM analysis was first performed within the standard brain space with regressors identical to those in the univariate analysis. For each run, the classifier in each searchlight was trained using $\beta$ maps for $100 \%$ coherent trials. To calculate the training data, we used the third and later trials after cue presentation and eliminated data from the second trials, to avoid potential confounds derived from cue-related effect.

Next, from $\beta$ maps for switch and repeat trials (i.e., immediately after cue presentation), image data were extracted within the same searchlight and scanning run. Then, the trained classifier was tested for whether it correctly classified the performed task (motion or color tasks) based on the extracted data (i.e., switch or repeat trials) for each run. It is important to note that training and tested maps were independent. Then, classification performance was collected from all functional runs and averaged within participants for each searchlight. A linear kernel was used and the $\mathrm{C}$ parameter was set to 1.0 (default).

For group-level analysis, accuracy maps were first contrasted between switch and repeat trials, and the contrasted accuracy maps were collected from all participants. Then voxelwise group-mean tests were performed, and the significance was tested based on the permutation method (5000 permutations) implemented in randomize in FSL. To exclude brain regions that showed a classification accuracy below the chance level in either of the switch or repeat trials, voxels with $t$ values $<0.0$ (uncorrected) in the group-level accuracy map of a switch or repeat trial were masked out when testing statistical significance. In other words, tested voxels showed classification accuracy above the chance level for both the switch and repeat trials. 
Table 1. Brain regions showing significant signal increase in the contrast of switch versus repeat trials ${ }^{a}$

\begin{tabular}{|c|c|c|c|c|c|}
\hline Area & $x$ & $y$ & $z$ & $z$ value & $B A$ \\
\hline \multirow[t]{22}{*}{ Frontal cortex } & 28 & 0 & 60 & 6.42 & 6 \\
\hline & 0 & 12 & 46 & 5.95 & 6 \\
\hline & -8 & 24 & 34 & 5.62 & 32 \\
\hline & 6 & 20 & 32 & 5.61 & 32 \\
\hline & -48 & 6 & 32 & 5.41 & 6 \\
\hline & -30 & -2 & 58 & 5.35 & 6 \\
\hline & -2 & 8 & 60 & 5.34 & 6 \\
\hline & 10 & 8 & 68 & 4.76 & 6 \\
\hline & -20 & 10 & 62 & 4.72 & 6 \\
\hline & -44 & 2 & 48 & 4.69 & 6 \\
\hline & -44 & 40 & 12 & 4.68 & 46 \\
\hline & -4 & 34 & 18 & 4.65 & $24 / 32$ \\
\hline & -42 & 28 & 22 & 4.59 & 46 \\
\hline & -24 & 8 & 46 & 4.43 & 6 \\
\hline & -8 & 0 & 72 & 4.38 & 6 \\
\hline & 26 & 40 & 26 & 4.2 & 9 \\
\hline & -58 & 14 & 18 & 4.13 & 44 \\
\hline & 46 & 18 & 10 & 3.78 & 44 \\
\hline & 28 & 52 & 26 & 3.63 & 10 \\
\hline & -50 & 6 & 10 & 3.55 & $6 / 44$ \\
\hline & -34 & -4 & 44 & 3.54 & 6 \\
\hline & 26 & -4 & 48 & 3.35 & 6 \\
\hline \multirow[t]{9}{*}{ Parietal cortex } & -54 & -30 & 44 & 5.66 & 40 \\
\hline & -44 & -40 & 50 & 5.63 & 40 \\
\hline & -8 & -70 & 50 & 4.93 & 7 \\
\hline & -34 & -52 & 46 & 4.89 & 39 \\
\hline & 16 & -70 & 52 & 4.73 & 7 \\
\hline & -28 & -70 & 36 & 4.62 & 39 \\
\hline & -58 & -26 & 30 & 4.61 & 40 \\
\hline & -44 & -40 & 32 & 4.24 & $39 / 40$ \\
\hline & -28 & -68 & 52 & 4.04 & 7 \\
\hline \multirow[t]{2}{*}{ Occipital cortex } & -18 & -66 & 26 & 5.02 & 19 \\
\hline & 16 & -66 & 34 & 4.68 & 19 \\
\hline \multirow[t]{7}{*}{ Others } & -32 & 14 & 2 & 5.64 & Insula \\
\hline & 28 & 24 & 6 & 5.07 & Insula \\
\hline & 36 & 10 & -2 & 4.76 & Insula \\
\hline & 28 & 20 & -8 & 4.67 & Insula \\
\hline & 30 & -68 & -52 & 4.31 & Cerebellum \\
\hline & 18 & -74 & -50 & 3.89 & Cerebellum \\
\hline & -44 & 10 & -2 & 3.68 & Insula \\
\hline
\end{tabular}

${ }^{a}$ Positive $z$ values indicate a signal increase in the switch trials. Coordinates are listed in the MNI space. BA, Brodmann areas (approximate).

ROI analyses were also performed for classification accuracy. ROIs in occipitotemporal regions were defined as being in the MT or VVC regions identified by univariate analysis (Fig. $2 B$; see Table 2). ROIs in the frontoparietal regions were independent of the current data to avoid circular analysis (Kriegeskorte et al., 2009) because, in the current study, the frontoparietal regions were identified based on switch and repeat trials involving perceptual uncertainty. Specifically, ROIs were defined based on the multiple demand network identified in a previous study (Camilleri et al., 2018). ROI images were created similarly to another ROI analysis for hemispheric laterality (see ROI analysis for hemispheric laterality), but separately for frontal and parietal regions. To test the robustness of the differential accuracy against the definition of ROI, we redefined ROIs based on the meta-analysis maps of Neurosynth, similarly to the analysis performed above. Then for each participant and ROI, classification accuracy was calculated for switch and repeat trials, and statistical significance was tested treating participants as a random effect.

Effective connectivity analysis. A dynamic causal modeling (DCM) (Friston et al., 2003) analysis was performed to examine functional network mechanisms associated with task switching during the perception of a motion or color stimulus. In particular, we hypothesized that
Table 2. Brain regions showing significant signal increase in the contrast of motion versus color trials ${ }^{a}$

\begin{tabular}{lrrrrl}
\hline Area & \multicolumn{1}{l}{$y$} & \multicolumn{1}{l}{$z$} & $z$ value & BA \\
\hline Frontal cortex & 40 & 38 & 12 & -4.11 & 46 \\
Parietal cortex & 2 & -24 & 62 & 3.8 & $4 / 6$ \\
& 26 & -60 & 42 & -5.61 & $7 / 39$ \\
& 28 & -68 & 52 & -4.56 & 7 \\
& -6 & -40 & 66 & 4.39 & 7 \\
& 58 & -26 & 20 & 4.33 & 40 \\
& -50 & -54 & 10 & 4.23 & 39 \\
& -28 & -62 & 50 & -4.23 & 7 \\
& -52 & -66 & 16 & 4.13 & 39 \\
& -54 & -34 & 34 & 4.02 & 40 \\
& -22 & -62 & 38 & -4.02 & 7 \\
& 32 & -34 & 44 & 3.86 & $1 / 40$ \\
& 36 & -36 & 60 & 3.84 & 1 \\
& 58 & -30 & 32 & 3.8 & 40 \\
& -30 & -70 & 32 & -3.41 & 39 \\
Tecipital cortex & -64 & -26 & 14 & 3.13 & $40 / 41$ \\
& -30 & -82 & 22 & -4.95 & 19 \\
& 32 & -72 & 18 & -4.72 & $19 / 39$ \\
& -42 & -64 & 8 & 4.83 & 19 \\
& 40 & -56 & 4 & 4.18 & $19 / 37$ \\
& 54 & -58 & 4 & 4.11 & 37 \\
& -34 & -54 & -16 & -3.98 & 37 \\
& 44 & -36 & 14 & 3.87 & 22 \\
& 56 & -40 & 12 & 3.83 & 22 \\
& -56 & -42 & 18 & 3.48 & 22 \\
\hline
\end{tabular}

${ }^{a}$ Positive $z$ values indicate a signal increase in motion trials, and negative $z$ values indicate a signal increase in color trials.

${ }^{\mathrm{b}}$ Corrected within a meta-analysis mask of a color-related region. The formats are similar to those in Table

functional connectivity among brain regions associated with (1) task switching, (2) perceptual decision-making, (3) motion perception, and (4) color perception is modulated by task manipulations and brain signals.

DCM allows us to explore effective connectivity among brain regions under the premise of the brain as a deterministic dynamic system that is subject to environmental inputs and produces outputs based on the space-state model. The model constructs a nonlinear system involving intrinsic connectivity, task-induced connectivity, and extrinsic inputs. Parameters of the nonlinear system are estimated based on fMRI signal (system states) and task events.

Four ROIs were first defined based on univariate activation contrasts:

1. Motion effect (MT; Fig. 3B) (Newsome and Pare, 1988; Britten et al., 1992, 1993; Shadlen et al., 1996; Beauchamp et al., 1997; Britten and Newsome, 1998; Braddick et al., 2001; Corbetta and Shulman, 2002; Huk et al., 2002; Mazurek et al., 2003; Kayser et al., 2010);

2. Color effect (VVC; Fig. 3B) (Zeki, 1973; Schein and Desimone, 1990; Zeki et al., 1991; Bartels and Zeki, 2000; Kayser et al., 2010);

3. Switching effect (lPFC; Fig. 3A) (Konishi et al., 1998, 2002; Dove et al., 2000; Rushworth et al., 2002; Braver et al., 2003; Crone et al., 2006; Brass and von Cramon, 2004; Jimura et al., 2004; Derrfuss et al., 2005; Yeung et al., 2006; C. Kim et al., 2011, 2012);

4. Perceptual uncertainty effect [right PFC (rPFC); Fig. 3C,D] (Kayser et al., 2010), with $6 \mathrm{~mm}$ radius spheres centered in the peak coordinates in group-level activation maps.

To avoid circular analysis (Kriegeskorte et al., 2009), the coordinates of ROIs were defined with a leave-one-subject-out procedure, where ROIs for 1 participant were determined based on other participants. ROIs in MT and VVC were defined based on a group-level statistical map for the contrast of motion and color trials with $100 \%$ coherence, respectively. IPFC ROI was defined based on a group-level statistical map for the contrast of switch and repeat trials. rPFC ROI was defined 
Table 3. Brain regions showing significant parametrical effect with motion coherence in motion trials ${ }^{a}$

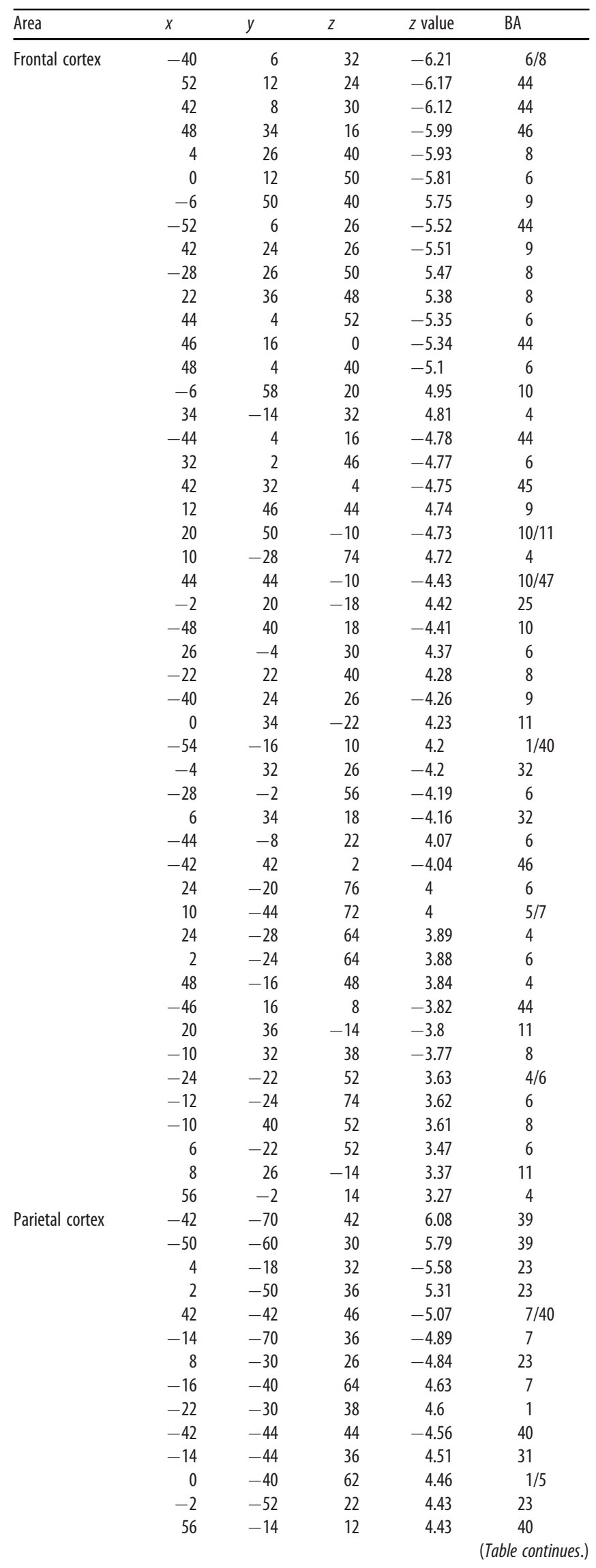

Table 3. Continued

\begin{tabular}{|c|c|c|c|c|c|}
\hline Area & $x$ & $y$ & $z$ & $z$ value & $B A$ \\
\hline & -54 & -32 & 42 & -4.43 & 40 \\
\hline & -58 & -28 & 28 & -4.37 & 40 \\
\hline & 54 & -30 & 40 & -4.33 & 40 \\
\hline & 22 & -44 & 68 & 4.31 & 7 \\
\hline & 50 & -60 & 28 & 4.13 & 39 \\
\hline & -24 & -34 & 24 & 4.09 & 40 \\
\hline & -26 & -60 & 44 & -4.07 & 7 \\
\hline & -12 & 30 & 14 & -4.02 & 24 \\
\hline & 8 & -36 & 34 & 4.01 & 23 \\
\hline & 30 & -48 & 44 & -4.01 & 7 \\
\hline & 22 & -46 & 56 & 3.97 & 7 \\
\hline & -4 & -36 & 72 & 3.89 & 5 \\
\hline & 24 & -32 & 50 & 3.65 & $1 / 5$ \\
\hline & -34 & -54 & 54 & -3.57 & 7 \\
\hline & 54 & -48 & 48 & -3.5 & 40 \\
\hline & -12 & -70 & 48 & -3.49 & 7 \\
\hline & 60 & 2 & -2 & 3.4 & 22 \\
\hline & -24 & -34 & 56 & 3.39 & 1 \\
\hline & -26 & -34 & 70 & 3.34 & 5 \\
\hline & 52 & -30 & 52 & -3.31 & 40 \\
\hline & -16 & -50 & 72 & 3.3 & 7 \\
\hline & 62 & -40 & 40 & -3.24 & 40 \\
\hline & -22 & -62 & 28 & -3.16 & $7 / 31$ \\
\hline \multirow[t]{11}{*}{ Temporal cortex } & -54 & -10 & -24 & 5.18 & 21 \\
\hline & -64 & -22 & -14 & 4.72 & 21 \\
\hline & 60 & -12 & -26 & 4.6 & 21 \\
\hline & -60 & 2 & -10 & 4.27 & 38 \\
\hline & -60 & 2 & -26 & 4.23 & 38 \\
\hline & -62 & -8 & 2 & 4.01 & 22 \\
\hline & -64 & -26 & 12 & 4.01 & 22 \\
\hline & 56 & 2 & -36 & 4 & 20 \\
\hline & -66 & -34 & 2 & 3.95 & 21 \\
\hline & -46 & -38 & -6 & 3.81 & 21 \\
\hline & -60 & -42 & -6 & 3.63 & 21 \\
\hline \multirow[t]{11}{*}{ Occipital cortex } & 2 & -74 & 4 & -4.82 & 18 \\
\hline & -4 & -86 & -2 & -4.62 & 18 \\
\hline & 12 & -78 & 12 & -4.61 & 17 \\
\hline & 16 & -64 & 36 & -4.49 & 19 \\
\hline & 24 & -84 & 40 & 4.24 & 19 \\
\hline & 10 & -82 & 0 & -4.07 & 17 \\
\hline & -12 & -48 & 0 & 3.83 & 19 \\
\hline & 12 & -88 & 34 & 3.73 & 19 \\
\hline & -10 & -70 & 8 & -3.29 & 17 \\
\hline & 10 & -94 & 8 & -3.26 & 17 \\
\hline & 0 & -66 & 22 & 3.25 & $18 / 31$ \\
\hline \multirow[t]{19}{*}{ Others } & -32 & 18 & 6 & -6.57 & Insula \\
\hline & 34 & 16 & 10 & -6.55 & 44/Insula \\
\hline & 32 & 22 & -4 & -5.99 & Insula \\
\hline & 0 & -48 & -32 & -5.8 & Cerebellum \\
\hline & -8 & 2 & 8 & -5.67 & Caudate \\
\hline & 12 & 8 & 16 & -5.54 & Caudate \\
\hline & 10 & -12 & 12 & -5.48 & Thalamus \\
\hline & -18 & -6 & -24 & 5.46 & Amygdala \\
\hline & 12 & 2 & 4 & -5.36 & Thalamus \\
\hline & -32 & -60 & -30 & -5.06 & Cerebellum \\
\hline & -26 & -14 & -16 & 4.9 & Hippocampus \\
\hline & -8 & -12 & 4 & -4.86 & Thalamus \\
\hline & -16 & -76 & -26 & -4.86 & Cerebellum \\
\hline & 22 & -10 & -20 & 4.78 & Hippocampus \\
\hline & 2 & -16 & -12 & -4.72 & Brainstem \\
\hline & 4 & -28 & -2 & -4.71 & Brainstem \\
\hline & -32 & -36 & -38 & -4.67 & Cerebellum \\
\hline & -20 & -70 & -50 & -4.64 & Cerebellum \\
\hline & 42 & -14 & 14 & 4.59 & $\begin{array}{l}\text { Insula } \\
\text { able continues. }\end{array}$ \\
\hline
\end{tabular}


Table 3. Continued

\begin{tabular}{rrrrrl}
\hline Area & \multicolumn{1}{l}{$y$} & \multicolumn{1}{l}{$z$} & $z$ value & BA \\
\hline & -20 & -60 & -30 & -4.27 & Cerebellum \\
16 & 8 & -8 & -4.12 & Putamen \\
32 & 6 & -6 & -3.88 & Putamen \\
& 2 & -60 & -28 & -3.84 & Cerebellum \\
& -16 & -18 & 18 & -3.47 & Thalamus \\
14 & -10 & -8 & -3.34 & Brainstem \\
\hline
\end{tabular}

${ }^{\text {a Positive } z \text { values indicate a signal increase in high-motion coherence trials, and negative } z \text { values indicate a }}$ signal increase in low-motion coherence trials. The formats are similar to those in Table 1.

based on a group-level statistical map for the coherence effect of switch and repeat trials.

Then, signal time courses of four ROIs and regressors in the events of interest were extracted from first-level GLMs. The events of interest were correct trials immediately after cue presentation (switch and repeat) and subsequent 100\% coherence trials. For switch and repeat trials, the contrast of the two trials and the normalized coherence level of stimuli were also added as a parametrical effect of interest. Nuisance effects of head motion, white matter signal, ventricle signal, functional run, and contrast were subtracted from the ROI time courses.

For each trial effect, causal models were defined as those that differed in external inputs and modulatory effects among ROIs. As the current analysis involved three ROIs, the tested models entailed 512 types (i.e., $2^{3}$ inputs and $2^{6}$ connection effects). Then, connectivity matrices reflecting (1) first-order connectivity, (2) effective change in coupling induced by the inputs, and (3) extrinsic inputs on the MRI signal in ROIs were estimated for each of the 512 models based on DCM analysis implemented in SPM12. A parametric regressor (switch vs repeat/coherence) was used as the extrinsic effect for effective connectivity between ROIs and ROI inputs.

To estimate the effective connectivity strength, a Bayesian model reduction method (Friston et al., 2016) was used. The reduction method enables the calculation of posterior densities of all possible reduced models, which was then inverted to a fully connected model. Then the reduced models were supplemented with second-level parametric empirical Bayes (Friston et al., 2016) to apply empirical priors that remove subject variability from each model.

Subsequently, parameters of these models were estimated based on Bayesian model averaging (Penny et al., 2010) to estimate connectivity changes. Because the current analysis aimed to identify the average effective connectivity observed across participants, we used a fixed-effect estimation assuming that every participant uses the same model, rather than a random-effect estimation assuming that different participants use different models, which has been often used to examine group differences in effective connectivity (Penny et al., 2010).

The significance of connectivity was then tested by thresholding at a posterior probability of $95 \%$ CI (uncorrected), as the current analysis aimed to test whether connectivity between two specific brain regions was enhanced depending on task manipulation and brain activity, but did not aim to explore one model involving connectivity among multiple brain regions that best fits to imaging and behavioral data.

Additionally, to test the robustness of the functional connectivity, we also examined Bayesian model averaging-based DCM without model reduction or without empirical prior, and confirmed the overall results were consistent across these estimation methods.

The number of trials for each condition (see Behavioral results) was comparable to those in recently published relevant studies examining cognitive control using DCM $(\sim 16-40$ trials for each condition) (Nee and D'Esposito, 2016; He et al., 2019; Bowling et al., 2020). We used a high temporal resolution sequence for functional imaging (Moeller et al., 2010) ( $\mathrm{TR}=0.8$; see also Imaging procedures), which is $>2$ times higher than these recent studies (TR $=$ 1.9-2.0 s), enabling us to collect more scan frames to increase the signal-to-noise ratio of the DCM estimation (Penny et al., 2004; Stephan et al., 2010).
Table 4. Brain regions showing significant parametrical effect with color coherence in color trials ${ }^{\mathrm{a}}$

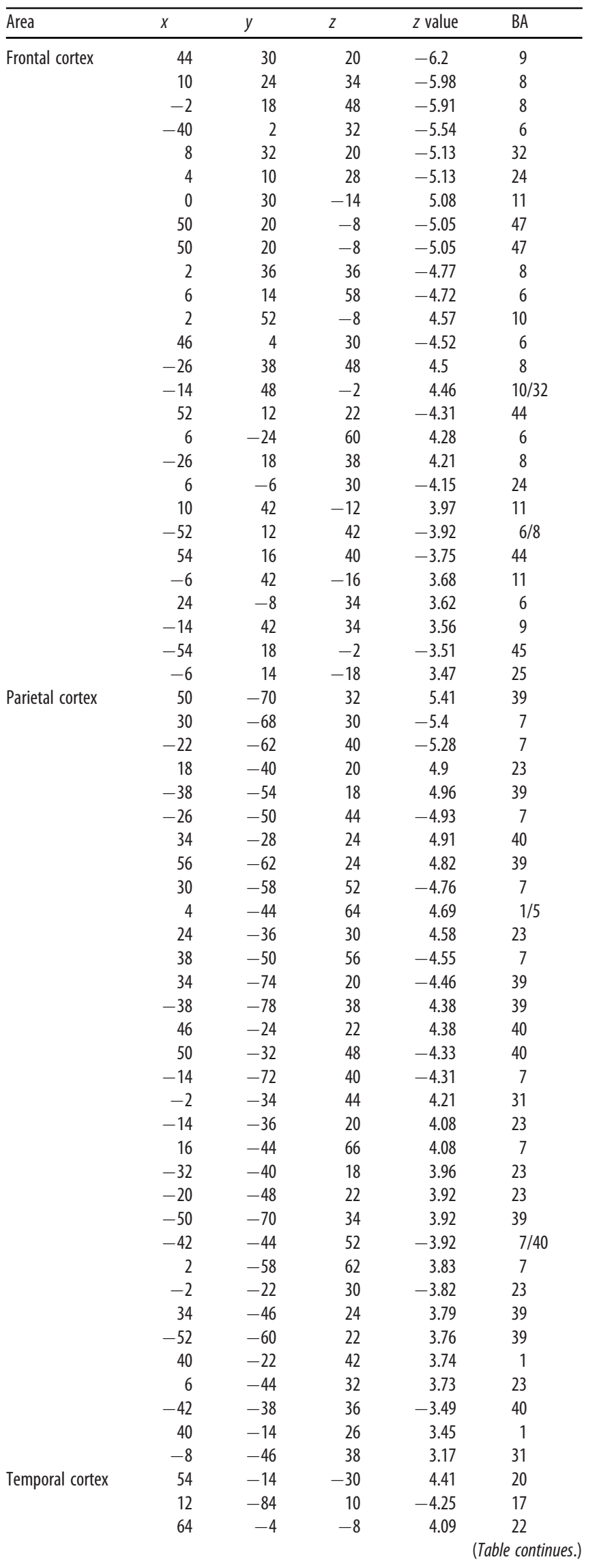


Table 4. Continued

\begin{tabular}{|c|c|c|c|c|c|}
\hline Area & $x$ & $y$ & $z$ & $z$ value & $B A$ \\
\hline & 58 & -8 & -20 & 4.08 & 21 \\
\hline & 60 & -12 & 0 & 4.01 & $22 / 41$ \\
\hline & 8 & -52 & 16 & 4 & 23 \\
\hline & 32 & -36 & 14 & 3.98 & 22 \\
\hline & -58 & -64 & 12 & 3.97 & 39 \\
\hline & -24 & -20 & -20 & 3.76 & 36 \\
\hline & 20 & -24 & -6 & -3.64 & 36 \\
\hline \multirow[t]{7}{*}{ Occipital cortex } & -30 & -78 & 22 & -5.03 & 19 \\
\hline & -8 & -86 & -2 & -4.05 & 18 \\
\hline & -2 & -72 & 0 & -3.93 & 18 \\
\hline & 60 & -60 & 8 & 3.92 & 37 \\
\hline & -10 & -52 & 10 & 3.86 & 23 \\
\hline & -12 & -74 & 14 & -3.74 & 17 \\
\hline & 12 & -66 & 8 & -3.61 & 17 \\
\hline \multirow[t]{22}{*}{ Others } & 32 & 24 & -2 & -6.51 & Insula \\
\hline & -10 & -76 & -22 & -6.44 & Cerebellum \\
\hline & -32 & 22 & 0 & -6.1 & Insula \\
\hline & -8 & -12 & -2 & -5.66 & Thalamus \\
\hline & -28 & -66 & -26 & -5.61 & Cerebellum \\
\hline & 36 & 16 & 6 & -5.52 & Insula \\
\hline & -10 & 4 & 4 & -5.3 & Caudate \\
\hline & 8 & -8 & 8 & -5.26 & Thalamus \\
\hline & 6 & -22 & -2 & -5.23 & Thalamus \\
\hline & 0 & -50 & -34 & -5 & Cerebellum \\
\hline & 8 & 4 & 4 & -4.99 & Thalamus \\
\hline & -20 & -8 & -18 & 4.89 & Amygdala \\
\hline & 10 & -76 & -26 & -4.77 & Cerebellum \\
\hline & 4 & -20 & -16 & -4.69 & Brainstem \\
\hline & 28 & -10 & 16 & 4.39 & Putamen \\
\hline & -36 & -46 & -34 & -4.38 & Cerebellum \\
\hline & 4 & -62 & -28 & -4.26 & Cerebellum \\
\hline & -40 & -66 & -30 & -4.03 & Cerebellum \\
\hline & 18 & 2 & 24 & 3.85 & Caudate \\
\hline & 22 & 14 & 18 & 3.84 & Caudate \\
\hline & -12 & -20 & 18 & -3.78 & Thalamus \\
\hline & 20 & -78 & -34 & -3.52 & Cerebellum \\
\hline
\end{tabular}

${ }^{\text {a Positive } z \text { values indicate a signal increase in high-color coherence trials, and negative } z \text { values indicate a }}$ signal increase in low-color coherence trials. The formats are similar to those in Table 1.

\section{Results}

\section{Behavioral results}

Participants gave correct responses for $35.56 \pm 4.90$ (mean \pm SD) switch trials and $38.70 \pm 6.41$ repeat trials in the motion task; in the color task, they gave correct responses for $36.07 \pm$ 3.74 switch trials and $33.70 \pm 4.68$ repeat trials. In the motion task, participants gave correct responses for $27.89 \pm 2.56$ highcoherence trials, $25.26 \pm 4.53$ middle-coherence trials, and $21.11 \pm 5.06$ low-coherence trials; in the color task, they gave correct responses for $23.30 \pm 2.97$ high-coherence trials, $25.63 \pm$ 3.54 middle-coherence trials, and $20.85 \pm 3.27$ low-coherence trials.

Accuracy became lower in the lower coherent (i.e., more uncertain) trials $\left(F_{(1,26)}=149.4, p<0.001, \eta^{2}=0.85\right)$, and became lower in switch trials than in repeat trials $\left(F_{(1,26)}=22.8\right.$; $p<0.001, \eta^{2}=0.47$ ) (Fig. $2 A$ ). The interaction effect of trial type (switch/repeat) and coherence levels was not significant $\left(F_{(1,26)}=2.1, p=0.16, \eta^{2}=0.08\right)$. Because dots were presented in the periphery visual field (see Materials and Methods), participants needed a higher signal-to-noise ratio compared with the centrally presented dots used in the previous studies (e.g., Palmer et al., 2005; Gold and Shadlen, 2007). This seems to be a major reason why the accuracy levels observed in this study were within the usual range despite very high coherence levels. Accordingly, reaction times became longer in lower coherent trials $\left(F_{(1,26)}=126.1 ; p<0.001, \eta^{2}=0.83\right)$, and became longer in switch trials than in repeat trials $\left(F_{(1,26)}=19.9 ; p<0.001, \eta^{2}=\right.$ $0.43)$ (Fig. $2 B$ ). The interaction effect of trial type (switch/repeat) and coherence levels was not significant $\left(F_{(1,26)}=3.9, p=0.058\right.$, $\eta^{2}=0.13$ ). These behavioral results suggest that the current behavioral task successfully manipulated task switching (Dove et al., 2000; Braver et al., 2003; Crone et al., 2006; Jimura et al., 2014) and perceptual decision-making (Shadlen et al., 1996; J. N. Kim and Shadlen, 1999; Mazurek et al., 2003; Palmer et al., 2005). In $100 \%$ coherent trials, reaction times also became longer in the color task than in the motion task $\left(F_{(1,26)}=10.1, p<0.005\right.$, $\left.\eta^{2}=0.28\right)$.

\section{Imaging results: univariate activation}

We first explored brain regions associated with task switching, motion perception, and color perception.

Figure $3 A$ shows brain regions with a significant increase and decrease in brain activity during task switching (switch trials vs repeat trials). Robust activation increases were observed in the left frontal regions, including the inferior frontal cortex (IFC), the dorsolateral PFC (DLPFC), the anterior PFC (aPFC), the inferior frontal junction (IFJ), and the pre-supplementary motor area (pre-SMA), and in the left parietal regions including the posterior parietal cortex (PPC). These left hemispheredominant frontoparietal activations are consistent with previous studies of task switching (Konishi et al., 1998, 2002; Dove et al., 2000; Rushworth et al., 2002; Braver et al., 2003; Crone et al., 2006; Brass and von Cramon, 2004; Jimura et al., 2004; Asari et al., 2005; Derrfuss et al., 2005; Yeung et al., 2006; C. Kim et al., 2011, 2012). A full list of brain regions is shown in Table 1.

Next, we explored brain regions associated with motion and color tasks during the task switching and repeat trials (Fig. 3B; Table 2). Activity became greater in the MT area during motion tasks, whereas in color tasks, greater activity was observed in VVC (Glasser et al., 2016). These results are also consistent with prior studies examining perceptual decision-making for motion (Newsome and Pare, 1988; Britten et al., 1992, 1993; Shadlen et al., 1996; Beauchamp et al., 1997; Britten and Newsome, 1998; Braddick et al., 2001; Corbetta and Shulman, 2002; Huk et al., 2002; Mazurek et al., 2003; Kayser et al., 2010) and color (Zeki, 1973; Schein and Desimone, 1990; Zeki et al., 1991; Motter, 1994; Bartels and Zeki, 2000; Kayser et al., 2010).

We then examined coherence effect. Whole-brain activation maps for the coherence effect in motion and color tasks are shown in Figure $3 C$ and Figure $3 D$, respectively. In both the color and motion tasks, activations became greater in low coherent trials in multiple frontoparietal regions, including the IFC, DLPFC, aPFC, IFJ, pre-SMA, and PPC (Fig. 3C,D; Tables 3 and 4) (Kayser et al., 2010). Interestingly, in both tasks, the coherence effect in frontoparietal regions looked prominent, especially in the right hemisphere.

To test this possibility, we first examined conjunction and disjunction of univariate maps for switch effect and negative motion coherence effect (motor and color tasks collapsed) $(p<0.05$ corrected; Fig. $4 A$ ). Frontoparietal regions in the right hemisphere showed the coherence effect predominantly, but the switch effect was almost absent in the right hemisphere. On the other hand, in the left frontoparietal regions, both of the switch and coherence effects were observed, but the switch effect showed more broadly. These results suggest a double dissociation in the frontoparietal 

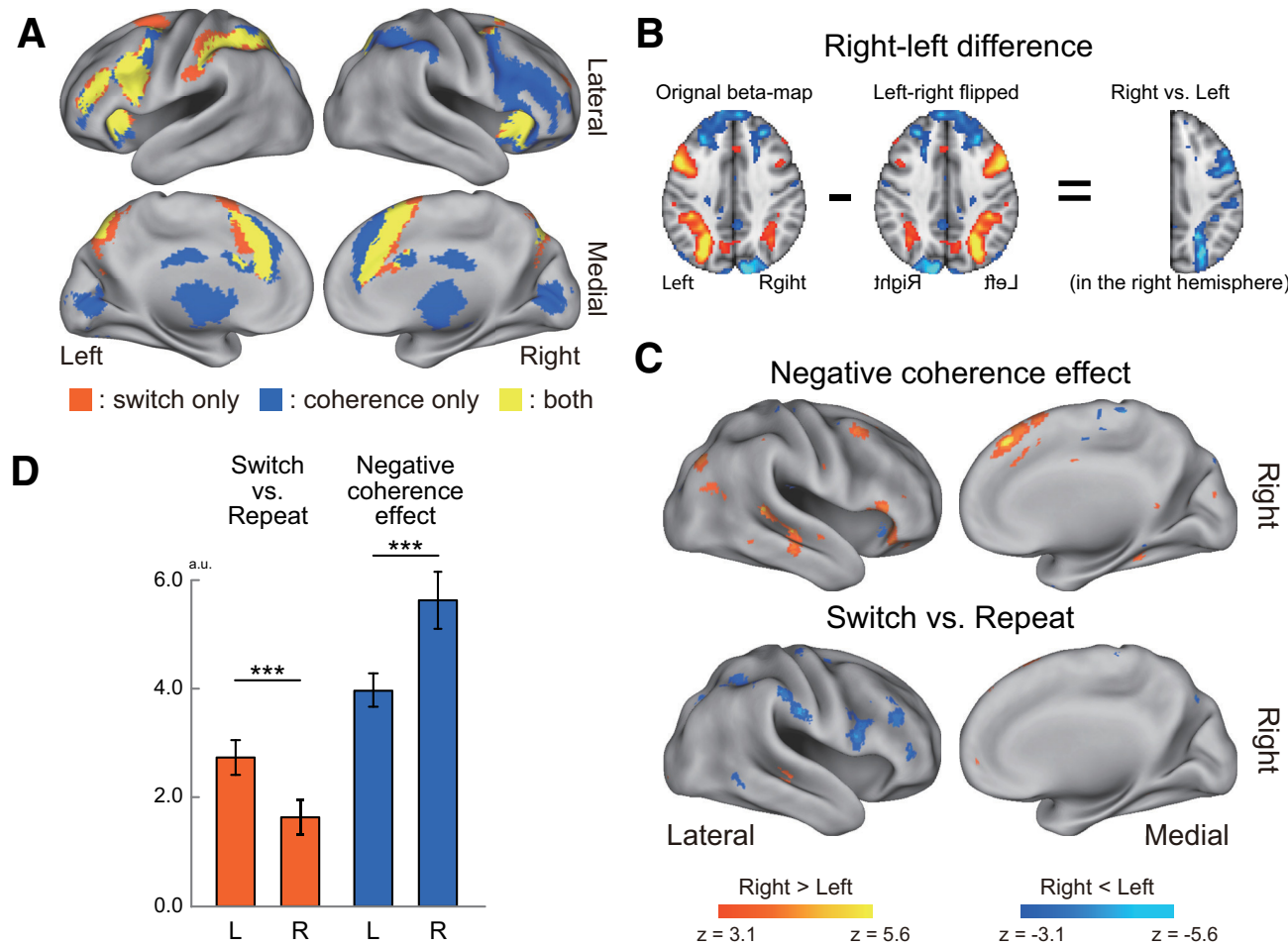

\section{C}

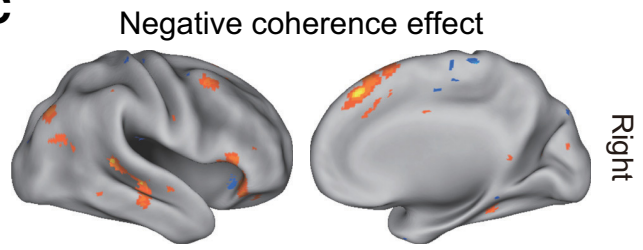

Switch vs. Repeat
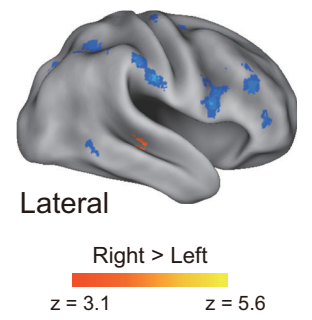
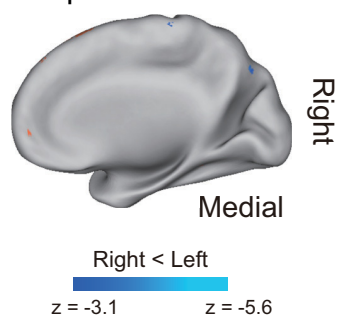

Figure 4. Hemispheric laterality. $\boldsymbol{A}$, Conjunction and disjunction maps. Statistical significance maps for switch and negative coherence effects $(p<0.05$ corrected across the whole brain) were mapped on the 3D surface image of the brain, and then color-coded. Orange represents switch effect only. Blue represents negative coherence effect only. Yellow represents both of switch and negative coherence effects. $\boldsymbol{B}$, Exploratory analysis procedure for hemispheric asymmetry. Contrast maps were flipped along the $x$ axis (left-right), then subtracted from the original nonflipped maps before being entered into voxelwise group-level analyses. C, Statistical map of brain regions showing a differential coherence effect between the right and left hemispheres (top). Hot colors represent a greater signal in low-coherent trials in the right hemisphere and/or a lower signal in high-coherent trials in the left hemisphere. Cool colors represent the reverse effect. Statistical map of brain regions showing differential activation for switch versus repeat trials between the right and left hemispheres (bottom). Hot colors represent greater activation for switch versus repeat trials in the right hemisphere and/or smaller activation for switch versus repeat trials in the left hemisphere. Cool color represents the reverse effect. Formats are similar to those in $\boldsymbol{B}$. D, ROI analysis. ROls in the frontoparietal cortex were defined independently of the current data, and then switch and negative coherence effects were compared between the right and left hemispheres. $* * * p<0.001$. L, Left; $R$, right.

regions, with switch and coherence effects involving the left and right frontoparietal regions, respectively.

Then, we explored brain regions to show the double dissociation. We contrasted the maps of the coherence effect between the right and left hemispheres on a voxel-by-voxel basis by flipping maps along the $x$ axis, and explored the brain regions showing a differential coherence effect between hemispheres by calculating group-level statistics (Fig. 4B; see also Materials and Methods). Figure $4 C$ (top) and Table 5 show brain regions with the differential effect. Positive effects were observed in multiple frontoparietal regions, including the DLPFC, pre-SMA, and PPC. Technically, a positive effect indicates greater activation in lowcoherent trials in the right hemisphere, and/or lower activation in high-coherent trials in the left hemisphere. These positive hemispheric laterality effects can be considered to be derived from greater activation in low-coherent trials in the right hemisphere, which was weaker in the contralateral (left) hemisphere, given the prominent coherence effects (greater activations in low-coherence trials) in those frontoparietal regions in the right hemisphere (Fig. 3C,D; Tables 3 and 4).

Similarly, to confirm the left-lateralized switch effect in frontoparietal regions, we contrasted the maps of the switching effect (switch vs repeat) between the right and left hemispheres. Figure $4 C$ (bottom) and Table 6 show a prominent negative effect in multiple frontoparietal regions, including the IFC, DLPFC, aPFC, IFJ, and PPC. Technically, again, a negative effect indicated greater switch-related activation in the left hemisphere and/or weaker switch-related activation in the right hemisphere; however, given the greater switch related activation in the left hemisphere (Fig. 3; Table 1), the negative effects were derived from greater switch-related activity in the left hemisphere, which was weaker in the contralateral hemisphere. This result was consistent with prior studies (Konishi et al., 2002; Jimura et al., 2004).

Finally, we performed ROIs analysis to further demonstrate the double dissociation. Frontoparietal ROIs in the left and right hemispheres were defined based on a previous study (Camilleri et al., 2018) (see also Materials and Methods); and for each of the left and right ROIs, the estimation contrast of the switch effect (switch vs repeat) and the parametric effect of the coherence were extracted for each participant. The switch effect was significant in both hemispheres (left: $t_{(26)}=8.5, p<0.001, r^{2}=0.74$; right: $\left.t_{(26)}=5.2, p<0.001, r^{2}=0.51\right)$, but was greater in the left hemisphere (left $>$ right: $t_{(26)}=5.3, p<0.001, r^{2}=0.52$ ) (Fig. $4 D$ ). The negative coherence effect (greater signal in low coherence trial) was also significant in both hemispheres (left: $t_{(26)}=12.8, p<0.001, r^{2}=0.86$; right: $t_{(26)}=10.64 p<0.001, r^{2}=$ 0.81 ), but conversely, the coherence effect was greater in the right hemisphere (right $>$ left: $t_{(26)}=4.7, p<0.001, r^{2}=0.46$ ). To test the robustness of the hemispheric dissociations against the definition of ROIs, we redefined ROIs based on the meta-analysis maps (Neurosynth; see Materials and Methods) (Yarkoni et al., 2011). The switch effect was significant in both hemispheres (left: $t_{(26)}=8.5, p<0.001, r^{2}=0.74$; right: $t_{(26)}=5.5, p<0.001, r^{2}$ 
Table 5. Brain regions showing significant difference in the low-coherence effect for the right hemisphere versus left hemisphere in switch and repeat trials $^{\mathrm{a}}$

\begin{tabular}{|c|c|c|c|c|c|}
\hline Area & $x$ & $y$ & $z$ & $z$ value & $B A$ \\
\hline \multirow[t]{14}{*}{ Frontal cortex } & 0 & 28 & 42 & 6.98 & 8 \\
\hline & 0 & 12 & 52 & 6.01 & 6 \\
\hline & 8 & 26 & 54 & 5.10 & 8 \\
\hline & 0 & 36 & 24 & 4.89 & 32 \\
\hline & 38 & 6 & 52 & 4.84 & $6 / 8$ \\
\hline & 44 & 26 & 2 & 4.55 & 45 \\
\hline & 10 & -14 & 50 & -4.53 & 6 \\
\hline & 10 & 40 & 36 & 4.31 & 9 \\
\hline & 48 & 24 & -10 & 4.29 & 47 \\
\hline & 14 & 20 & 44 & 4.25 & 8 \\
\hline & 10 & 16 & 62 & 4.19 & 6 \\
\hline & 30 & -14 & 58 & -4.18 & 6 \\
\hline & 52 & 22 & 30 & 4.14 & 44 \\
\hline & 34 & -8 & 46 & -3.44 & 6 \\
\hline \multirow[t]{5}{*}{ Parietal cortex } & 36 & -70 & 34 & 4.71 & 39 \\
\hline & 38 & -54 & 18 & 4.70 & 39 \\
\hline & 40 & -74 & 46 & 4.65 & 39 \\
\hline & 48 & -76 & 30 & 4.22 & 39 \\
\hline & 48 & -44 & 54 & 3.95 & 40 \\
\hline \multirow[t]{3}{*}{ Temporal cortex } & 50 & -26 & -2 & 4.76 & 22 \\
\hline & 48 & -40 & 10 & 4.67 & 22 \\
\hline & 60 & -24 & -12 & 4.34 & 21 \\
\hline \multirow[t]{3}{*}{ Occipital cortex } & 0 & -82 & 0 & 4.53 & 18 \\
\hline & 14 & -82 & 12 & 4.45 & 17 \\
\hline & 42 & -70 & 20 & 3.45 & 19 \\
\hline \multirow[t]{6}{*}{ Others } & 0 & -48 & -34 & 5.91 & Cerebellum \\
\hline & 28 & -62 & -24 & -4.82 & Cerebellum \\
\hline & 30 & -66 & -56 & -4.73 & Cerebellum \\
\hline & 0 & -62 & -28 & 4.41 & Cerebellum \\
\hline & 34 & 20 & 8 & 3.84 & Insula \\
\hline & 42 & -62 & -42 & -3.75 & Cerebellum \\
\hline
\end{tabular}

${ }^{a}$ Positive $z$ values indicate a signal increase in the right hemisphere, and negative $z$ values indicate a signal increase in the left hemisphere. The formats are similar to those Table 1.

$=0.54)$, but was greater in the left hemisphere than the right hemisphere (left $>$ right: $t_{(26)}=3.7, p<0.01, r^{2}=0.34$ ). The negative coherence effect was also significant in both hemispheres (left: $t_{(26)}=9.8, p<0.01, r^{2}=0.79$; right: $t_{(26)}=10.7, p<0.001, r^{2}$ $=0.81$ ), but was greater in the right hemisphere (right $>$ left: $\left.t_{(26)}=6.4, p<0.001, r^{2}=0.61\right)$.

These collective results clearly demonstrate hemispheric asymmetries in the frontoparietal regions: task switching and perceptual decision-making are associated with the left and right hemispheres, respectively, suggesting distinctive neural mechanisms across hemispheres. No regions showed an interaction between the switch and coherence effects.

\section{Searchlight MVPA}

Given the brain regions associated with tasks to be performed, switching tasks, and perceptual demand identified by univariate activation analysis, we next explored brain regions involving task-related neural representation that may reflect appropriate neural coding of the task to be performed. More specifically, brain activity patterns were examined if the pattern involved discriminable information of motion and color tasks based on searchlight MVPA (Kriegeskorte et al., 2006). Importantly, in the current analysis, training and test data were independent; training and test data were based on $100 \%$ coherent trials and switch/ repeat trials, respectively (Fig. $1 B$; see also Materials and Methods).
Table 6. Brain regions showing significant difference for the right hemisphere versus left hemisphere in contrast of switch versus repeat trials ${ }^{a}$

\begin{tabular}{|c|c|c|c|c|c|}
\hline Area & $x$ & $y$ & $z$ & $z$ value & $B A$ \\
\hline \multirow[t]{13}{*}{ Frontal cortex } & 0 & 12 & 46 & 5.94 & 6 \\
\hline & 0 & 24 & 32 & 5.37 & 32 \\
\hline & 0 & 8 & 60 & 5.28 & 6 \\
\hline & 44 & 42 & 10 & -5.17 & 46 \\
\hline & 48 & 6 & 18 & -4.59 & 44 \\
\hline & 46 & 8 & 36 & -4.52 & $6 / 8$ \\
\hline & 0 & 36 & 20 & 4.49 & 32 \\
\hline & 42 & 28 & 26 & -4.38 & 9 \\
\hline & 46 & 28 & 40 & -4.28 & 9 \\
\hline & 8 & 16 & 64 & 3.94 & 6 \\
\hline & 28 & -20 & 64 & -3.94 & 6 \\
\hline & 12 & 28 & 58 & 3.84 & 6 \\
\hline & 0 & 10 & 30 & 3.78 & 24 \\
\hline \multirow[t]{8}{*}{ Parietal cortex } & 56 & -22 & 30 & -5.02 & $1 / 40$ \\
\hline & 28 & -70 & 36 & -5.00 & 39 \\
\hline & 56 & -30 & 44 & -4.68 & 40 \\
\hline & 34 & -52 & 46 & -4.58 & 39 \\
\hline & 46 & -44 & 46 & -4.47 & 40 \\
\hline & 10 & -76 & 38 & -4.30 & 7 \\
\hline & 18 & -76 & 56 & -3.99 & 7 \\
\hline & 38 & -34 & 36 & -3.96 & $1 / 40$ \\
\hline \multirow[t]{2}{*}{ Temporal cortex } & 50 & -30 & -8 & 4.17 & 21 \\
\hline & 58 & -58 & -6 & -4.00 & 37 \\
\hline Occipital cortex & 30 & -72 & 20 & -3.64 & 19 \\
\hline Others & 30 & -70 & -48 & 4.15 & Cerebellum \\
\hline
\end{tabular}

${ }^{a}$ The formats are similar to those in Table 5 .

Occipitotemporal regions showed greater accuracy in the switch trials (Fig. $5 A$, left) but not in the repeat trials (Fig. $5 A$, middle). Then, to identify brain regions showing enhanced task coding during task switching, classification accuracy maps were contrasted between switch and repeat trials on a voxel-by-voxel basis (Jimura et al., 2014); and a group-level analysis was performed. The occipitotemporal regions showed a differential effect (Fig. 5A, right; Table 7). As stated above, in this region, there is a greater amount of information about performed tasks in switch trials relative to repeat trials. Of interest, this region is located spatially between the MT region (showing greater activity during motion tasks; Fig. 5B, blue) and the VVC region (showing greater activity during the color task; Fig. $5 B$, green), which were identified in a univariate activation analysis (Fig. 2B; Table 2). Thus, the occipitotemporal region adjacent to the stimulus-modality-dependent MT/VVC areas showed enhanced task coding during task switching. It is possible that enhanced task coding in this middle region reflects enhanced task coding in the MT/VVC areas during task switching (see Discussion). To examine this possibility, an ROI analysis was performed. ROIs were defined as MT and VVC regions that were identified by univariate analysis (Fig. 2B; Table 2); the classification accuracy was averaged for each of the ROIs for switch and repeat trials. The MT region showed greater classification accuracy in both the switch and the repeat trials than the chance level (switch: $t_{(26)}=6.3, p<0.001, r^{2}$ $=0.60$; repeat: $\left.t_{(26)}=3.1, p<0.01, r^{2}=0.27\right)$, but the accuracy was greater in the switch than in the repeat trial $\left(t_{(26)}=5.8\right.$; $\left.p<0.001, r^{2}=0.42\right)$. In the VVT region, classification accuracy was greater in the switch trial $\left(t_{(26)}=7.1, p<0.001, r^{2}=0.66\right)$, but not in the repeat trial $\left(t_{(26)}=-0.2, p=0.83, r^{2}=0.00\right)$, compared with the chance level. The accuracy difference was also significant $\left(t_{(26)}=4.7, p<0.001, r^{2}=0.46\right)$

We also performed an ROI analysis for the frontoparietal regions. ROI images were created similarly to the hemispheric 
A

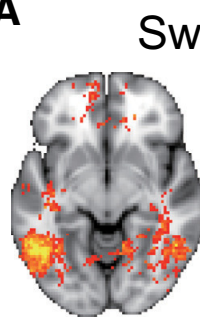

$Z=-8$
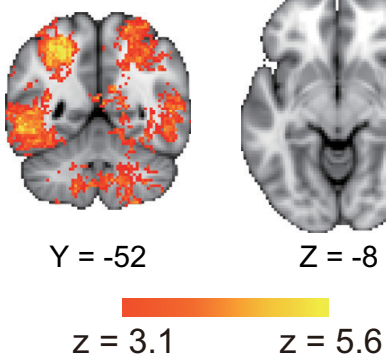

Repeat

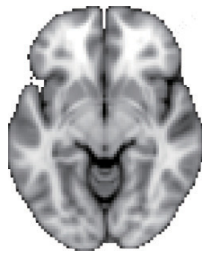

$z=5.6$

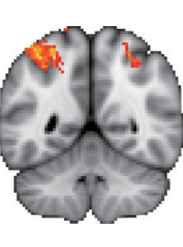

$Y=-52$
Switch vs. Repeat

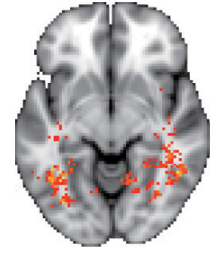

$Z=-8$

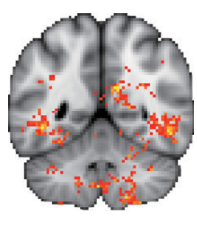

$Y=-52$
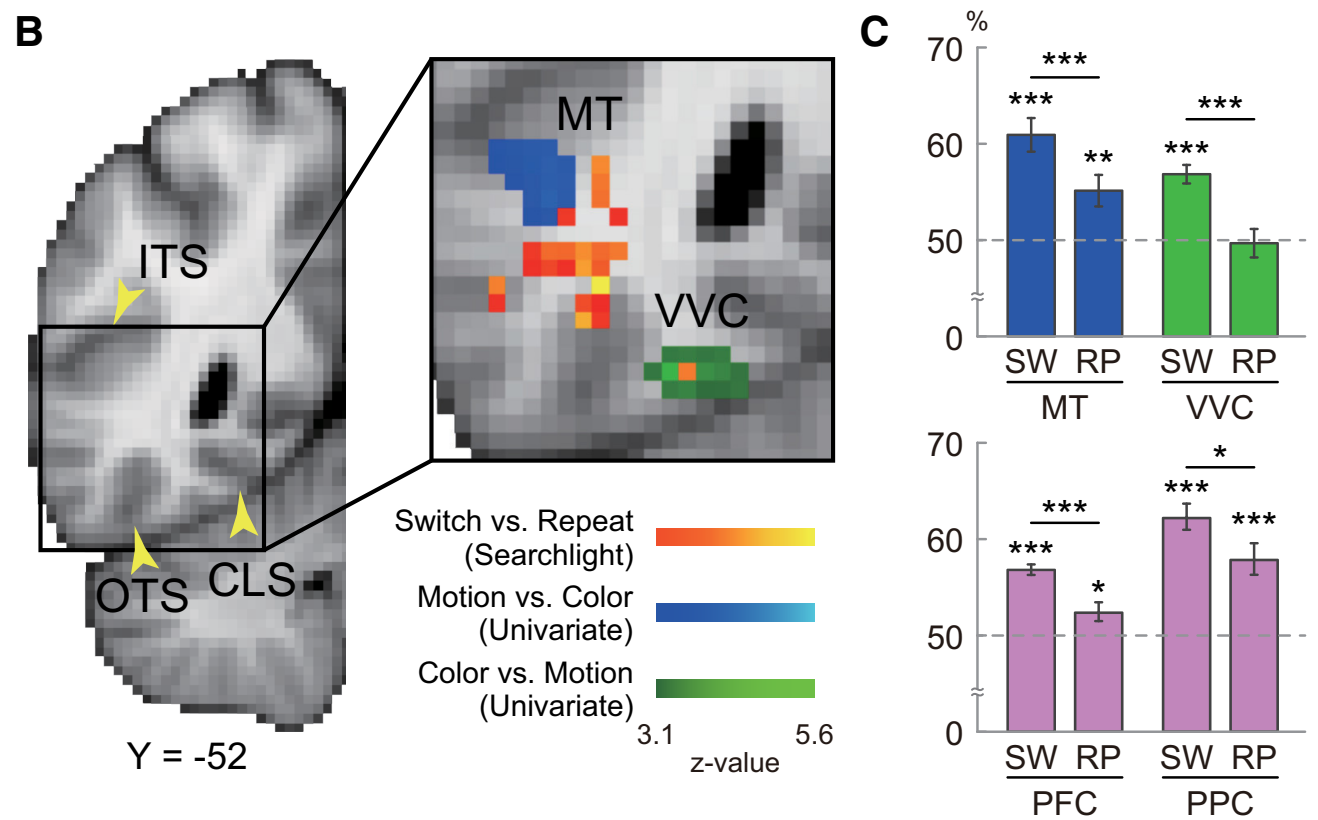

Figure 5. Searchlight MVPA. A, Statistical significance maps of classification accuracy for motion and color tasks in searchlight MVPA. Switch trial (left), repeat trial (middle), and switch versus repeat trials (right). Hot colors represent greater accuracy in switch and repeat trials (relative to chance; left and middle) and in switch relative to repeat trials (right). Cool colors represent the reverse effect. $\boldsymbol{B}$, The statistical maps were overlaid on a $2 \mathrm{D}$ section as indicated by the $y$ axis on the left. Black solid square on the section (left) represents the area magnified on the right. Hot colors represent brain regions showing greater classification accuracy for switch trials relative to repeat trials in the searchlight MVPA. Cool and green colors represent brain regions with greater activation during motion relative to color tasks, and during color relative to motion tasks in univariate analyses, respectively. Yellow arrowheads indicate major anatomic landmarks on the $2 \mathrm{D}$ section at left. ITC, Inferior temporal sulcus; OTS, occipitotemporal sulcus; CLS, collateral sulcus. C, ROI analysis. For switch (SW) and repeat (RP) trials, voxelwise accuracy was averaged within MT and VVC identified by the univariate analysis in $\boldsymbol{A}$ (top), and also within the PFC and PPC ROls defined independently of the current data (bottom). Error bars indicate SEM across participants. $* p<0.05 ; * * p<0.01 ; * * * p<0.001$.

laterality analysis, but for the frontal and parietal regions, separately (Fig. 4D; see also Materials and Methods). In the frontal ROI, the accuracy was higher than the chance level in the switch and repeat trials (switch: $t_{(26)}=9.0, p<0.001, r^{2}=0.75$; repeat: $\left.t_{(26)}=2.3, p<0.05, r^{2}=0.17\right)$, but was higher in the switch trial than in the repeat trial (switch $>$ repeat: $t_{(26)}=4.7, p<0.001$, $r^{2}=0.45$ ). In the parietal ROI, the accuracy was higher than the chance level in the switch and repeat trials (switch: $t_{(26)}=8.4$, $p<0.001, r^{2}=0.72$; repeat: $\left.t_{(26)}=4.5, p<0.001, r^{2}=0.43\right)$, but was higher in the switch trial than in the repeat trial (switch $>$ repeat: $\left.t_{(26)}=2.3, p<0.05, r^{2}=0.17\right)$. To test the robustness of the differential accuracy against the definition of ROI, we redefined ROIs based on the meta-analysis maps of Neurosynth, similar to the analysis above. Consistent results were obtained in the frontal ROI (switch: $t_{(26)}=9.1, p<0.001, r^{2}=0.76$; repeat: $t_{(26)}=1.9, p=0.07, r^{2}=0.12$; switch $>$ repeat: $t_{(26)}=4.4, p<$ $0.001, r^{2}=0.43$ ) and parietal ROI (switch: $t_{(26)}=10.3, p<0.001$, $r^{2}=0.80$; repeat: $t_{(26)}=5.3, p<0.001, r^{2}=0.51$; switch $>$ repeat: $\left.t_{(26)}=3.7, p<0.001, r^{2}=0.35\right)$. These results suggest that the frontoparietal regions implicated in task switching and perceptual uncertainty also enhanced task coding in the switch relative to the repeat trials.

\section{Effective connectivity analysis}

The whole-brain exploratory analyses identified three types of brain regions: (1) occipitotemporal MT and VVC regions associated with motion and color perception, respectively; (2) IPFC associated with task switching; and (3) rPFC associated with perceptual uncertainty. In addition, searchlight MVPA identified an occipitotemporal region between MT and VVC where task information was increased during task switching. One possible mechanism to comprehensively explain these results is that the MT/ VVC regions received task-switching signals from the IPFC, and the task information was complemented by signaling from the rPFC when task-relevant information involved uncertainty. To test this hypothesis, we performed interregional effective 
connectivity analysis for switch and coherence effects during task switching based on DCM (Friston et al., 2003), which allowed us to examine the directionality of taskrelated functional connectivity via the state-space model (see also Materials and Methods).

As shown in Figure 6A, the connectivity was enhanced from IPFC toward MT in the switch-to-motion trials. By contrast, connectivity in the reverse direction (i.e., from MT toward $\mathrm{PFC}$ ) was enhanced in repeat-motion trials. On the other hand, in color tasks, the connectivity was enhanced from IPFC toward VVC in the switch-to-color trials, and the connectivity was enhanced in the opposite direction during the repeat-color trials (Fig. 6B). These results suggest that modality-specialized occipitotemporal regions receive and send signals toward the switchrelated prefrontal region, depending on whether the task was switched or repeated.

In low coherence motion trials, the connectivity was enhanced from rPFC toward MT, and from rPFC toward IPFC (Fig. $6 \mathrm{C}$ ). In contrast, in the high-coherence motion trials, the connectivity was enhanced from MT to $\mathrm{PFC}$ and $\mathrm{rPFC}$, and also from IPFC to rPFC. On the other hand, in the low-coherence color trials, the connectivity was also enhanced from rPFC toward VVC, from rPFC toward IPFC, and from V4 toward IPFC (Fig. 6D). In the high-coherence color trials, the connectivity was enhanced from IPFC toward VVC and rPFC. These results suggest that rPFC has a key role in signaling the switch-related IPFC and modality-specialized occipitotemporal regions when perceptual demand increases because of the increased the perceptual uncertainty.

Together, during task switching in a situation where external perceptual information involves more uncertainty, the top-down signal from the prefrontal regions associated with task switching and perceptual uncertainty becomes stronger toward the stimulus-modality-dependent occipitotemporal regions. On the other hand, the bottom-up signal from the occipitotemporal regions to the prefrontal region becomes stronger during repeat trials. These results collectively suggest complementary prefrontal mechanisms during task switching, with perceptual uncertainty with the IPFC and rPFC for task switching and perceptual decision-making, respectively. Then, the prefrontal mechanisms increase task-related information in the occipitotemporal regions.

\section{Discussion}

The current study examined neural mechanisms during task switching in a situation where goal-relevant information involved perceptual uncertainty. The IPFC, MT/VVC, and $\mathrm{rPFC}$ were associated with task switching, perception of the target stimulus of an engaged task, and perceptual demands, respectively. During task switching, the IPFC signaled to the MT and VVC regions, and task-related neural pattern information in the cortical region increased in an occipitotemporal region between MT and VVC. On the other hand, when goal-relevant information involved more perceptual uncertainty, the IPFC and MT/ VVC received a signal from the rPFC. These collective results suggest distributed cortical mechanisms during task switching under perceptual uncertainty, where cross-hemispheric prefrontal mechanisms complementarily signal to task-dependent perceptual regions in the occipitotemporal cortex (Fig. 7).

Behaviorally, the interaction effect of switching and coherence levels was not strong, as it failed to reach statistical significance. In contrast, both of the two main effects (switching and
Table 7. Brain regions showing a significant difference in accuracy to classify task dimension (motion/color) between the switch and repeat trials ${ }^{a}$

\begin{tabular}{crrrll}
\hline Area & \multicolumn{1}{c}{$x$} & $y$ & $z$ & $z$ value & BA \\
\hline Frontal cortex & -30 & 0 & 42 & 4.67 & $8 / 6$ \\
& -34 & -14 & 52 & 3.63 & 6 \\
& -26 & -4 & 54 & 3.56 & 6 \\
Parietal cortex & 34 & -68 & 24 & 4.69 & $19 / 39$ \\
& 42 & -76 & 20 & 4.37 & 19 \\
& 46 & -28 & 42 & 4.25 & 40 \\
& -40 & -82 & 22 & 4.10 & 19 \\
& -44 & -34 & 34 & 4.00 & 40 \\
& 58 & -30 & 34 & 3.99 & 40 \\
& -28 & -84 & 22 & 3.92 & 19 \\
& 22 & -74 & 30 & 3.79 & $19 / 39$ \\
& -26 & -70 & 14 & 3.63 & 19 \\
& -42 & -28 & 52 & 3.60 & 40 \\
& -34 & -32 & 44 & 3.47 & 40 \\
& 46 & -72 & 34 & 3.33 & 39 \\
& -28 & -40 & 32 & 3.29 & 40 \\
& -40 & -82 & 38 & 3.13 & $39 / 19$ \\
& -52 & -56 & -4 & 4.86 & 37 \\
& 50 & -52 & -10 & 4.65 & 37 \\
& -40 & -70 & 6 & 4.43 & 19 \\
& -34 & -56 & -16 & 3.91 & 37 \\
& -48 & -46 & 6 & 3.88 & $21 / 37$ \\
& -40 & -56 & -2 & 3.87 & $37 / 19$ \\
& -48 & -62 & -16 & 3.73 & 37 \\
& 56 & -52 & 2 & 3.51 & $37 / 21$ \\
& 42 & -58 & -2 & 3.45 & $19 / 37$ \\
& -40 & -68 & -8 & 3.38 & $37 / 19$ \\
\hline
\end{tabular}

${ }^{a}$ Positive $z$ values indicate higher accuracy in the switch trial. The formats are similar to those in Table 1.

coherence) were statistically reliable. In univariate imaging analyses, the two main effects were predominantly observed across the whole brain, but the interaction effect was absent. These behavioral and imaging results suggest that the main effects of switching and coherence are dominant. Thus, for putative mechanisms for successful task switching under perceptual uncertainty, the current study suggests that distinct mechanisms for perceptual decision-making and task switching cooperatively guide successful task switching. More specifically, when target information involves uncertainty, activation in the right prefrontal region becomes greater, and the cross-hemispheric signal from the right prefrontal region toward the left prefrontal region is enhanced (Fig. 7). These results may suggest that the perceptual decision-making mechanism involves the right frontoparietal regions and helps to achieve task switching involving the left frontoparietal regions.

Previous studies of task switching have focused on perceptual decision-making and demand, where the target stimulus involved uncertainty, and revealed occipitotemporal and frontoparietal mechanisms (Kayser et al., 2010; Mante et al., 2013; Zhang et al., 2013; Kumano et al., 2016). More recent studies examined task switching involving perceptual uncertainty where the relevant task was not explicitly cued and the switch to an alternative task was guided by feedback to the response (Purcell and Kiani, 2016), and identified medial prefrontal mechanisms critical for voluntary switching (Sarafyazd and Jazayeri, 2019). In contrast, the current study focused on cue-guided switching when a perceptually ambiguous stimulus was presented. Two neural mechanisms (one associated with task switching involving the IPFC and the other associated with perceptual decision-making involving the rPFC, MT, and VVC) cooperatively guide flexible adaptation to changing environments. 
A

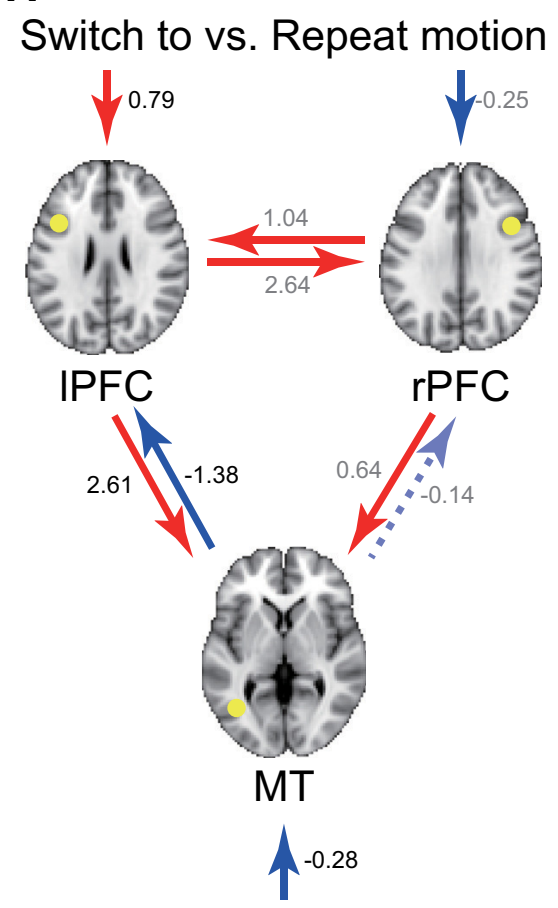

C

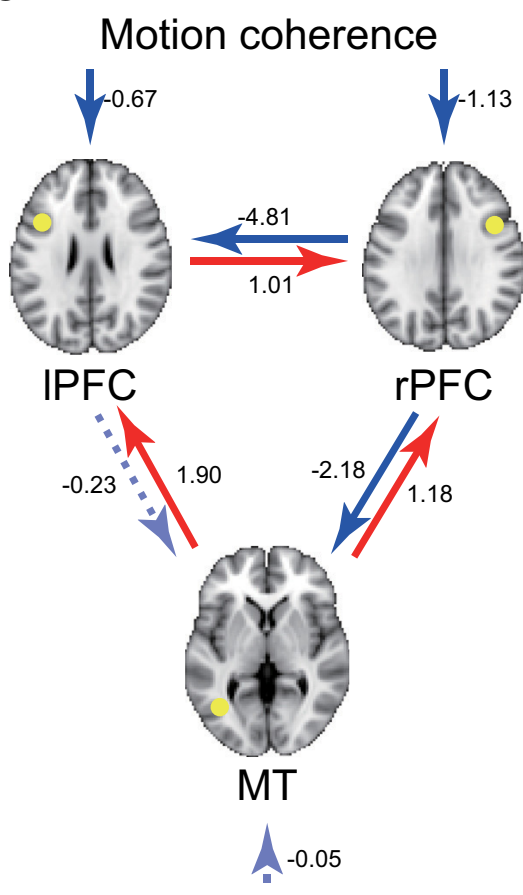

B

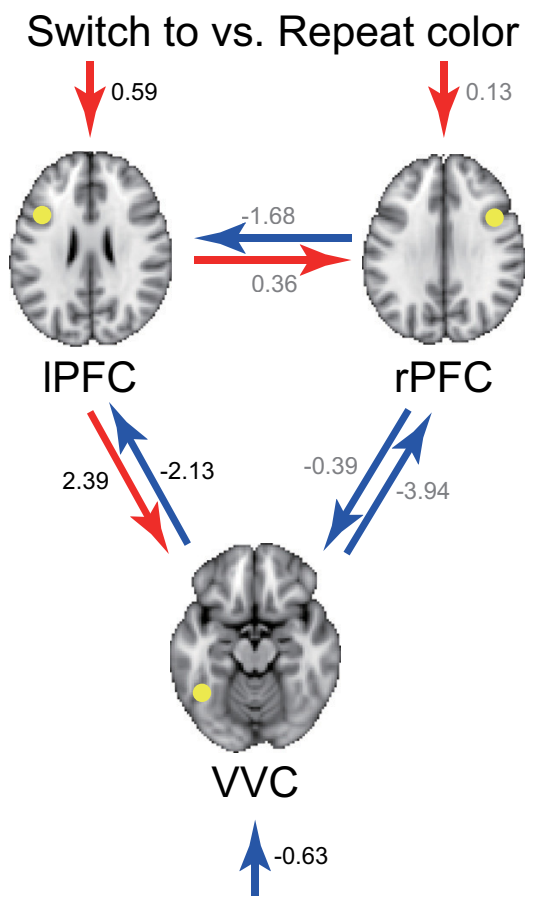

D

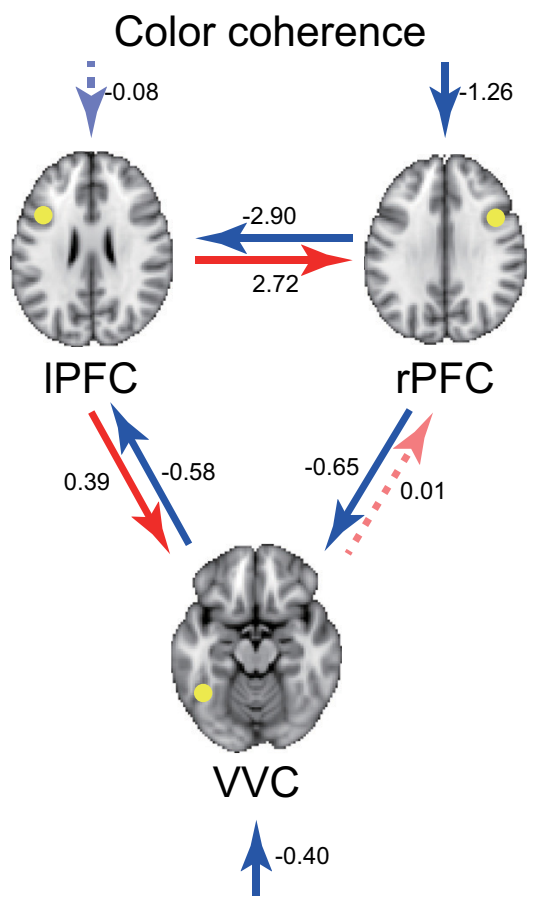

Figure 6. Effective connectivity analysis based on DCM. A, Effective connectivity and extrinsic inputs modulated in switch trials relative to repeat trials during motion tasks. Red arrows indicate enhancements of connectivity in switch trials. Blue arrows indicate enhancements of connectivity in repeat trials. Solid and dotted lines indicate effective connectivity that is statistically significant ( $P$ $<0.05$; uncorrected) and insignificant, respectively. Positive values indicate connectivity enhancements in switch trials. Negative values indicate connectivity enhancements in and repeat trials. $\boldsymbol{B}$, Effective connectivity and extrinsic inputs modulated in switch relative to repeat trials during color tasks. The formats are similar to those in $\boldsymbol{A}$. $\boldsymbol{C}$, Effective connectivity and extrinsic inputs modulated by motion coherence during motion tasks. Red arrows indicate enhancements in high-coherence trials. Blue arrows indicate enhancements in low-coherence trials. Solid and dotted lines indicate effective connectivity that was statistically significant $(p<0.05$; uncorrected) and insignificant, respectively. Positive values indicate connectivity enhancements in high-coherence trials. Negative values indicate connectivity enhancements in low-coherence trials. $\boldsymbol{D}$, Effective connectivity and extrinsic inputs modulated by color coherence during color tasks. The formats are similar to those in $\boldsymbol{C}$.

DCM analyses revealed a top-down signal from the IPFC to the MT and VVC regions during task switching, depending on the task that was switched. More specifically, the IPFC signaled to the MT during a switch from the color to motion tasks, and to
VVC during a switch from the motion to color tasks. This topdown mechanism may reflect supplemental attention to a visual stimulus required to collect task-related information to make a decision, with the prefrontal region complementing stimulus- 


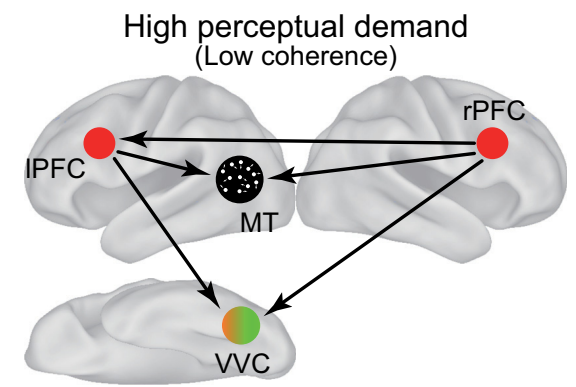

Low perceptual demand (High coherence)

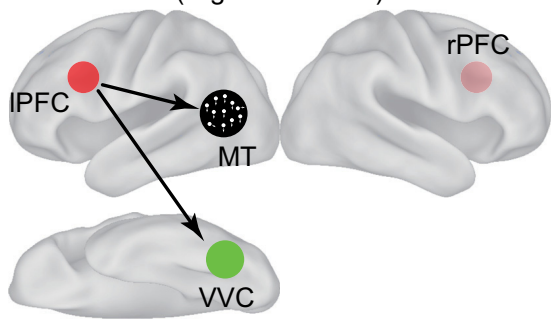

Figure 7. Schematic diagrams of neural mechanisms among the IPFC, rPFC, MT, and VVC during task switching with perceptual uncertainty. Arrows indicate signal directions.

modality-dependent perceptual information in the occipitotemporal region (Desimone and Duncan, 1995; Tomita et al., 1999; Kastner and Ungerleider, 2000; Miller and Cohen, 2001; Corbetta and Shulman, 2002). The current study extends this view that the top-down signal from the prefrontal to occipitotemporal regions is enhanced when the stimulus involves perceptual uncertainty, and the enhanced prefrontal signal was supplemented from the contralateral hemisphere (Fig. 7).

A searchlight MVPA analysis identified an occipitotemporal region showing enhanced task coding in the middle of the MT and VVC. It can be speculated that, during task switching, task coding was enhanced in the MT and VVC regions, which reciprocally increased the amount of discriminable task information in this middle region, which is consistent with the follow-up ROI analysis (Fig. 5B). This interpretation is also compatible with DCM results showing an enhanced top-down signal from the IPFC to the MT or VVC depending on the task to be switched. It is well known that occipitotemporal regions involve stimulusmodality specialized patchy regions (e.g., Shadlen and Newsome, 1994; Treisman and Kanwisher, 1998). The exploratory searchlight MVPA (Fig. 5A) result is dependent on these patchy characteristics; and for this result to occur, it may be important for the MT and VVC to be located relatively close ( $\sim 25 \mathrm{~mm}$ apart; Table 2) within the occipitotemporal region.

In the current study, the decoding accuracy in the occipitotemporal regions may be lower for visual sensory regions. One possible reason for this is that the visual stimulus was identical in the motion and color tasks, which may have attenuated the sensitivity of visual cortical areas that are sensitive to visual evidence. Another possibility is that the classifier was not optimized to classify the trials immediately after cue presentation involving perceptual uncertainty because the classifier was trained based on $100 \%$ coherence trials that do not involve perceptual uncertainty.

A recent MVPA study of task switching suggested that task coding in frontoparietal regions is attenuated in a switch trial (Qiao et al., 2017), whereas another study suggested that task coding is independent of task switching (Loose et al., 2017). On the other hand, in the current study, the classification accuracy in the frontoparietal regions was greater in the switch trial. The variability of accuracy patterns in the previous and current studies may be because of the differences in behavioral design and cognitive demands of task switching. In previous studies, a taskcueing paradigm was used, where a task cue was presented in each trial (Loose et al., 2017; Qiao et al., 2017); conversely, the current study used an intermittent cue paradigm in which the switch trial occurred after successive correct trials for the alternative task without presenting a cue (Fig. 1; see also Materials and Methods). In the latter paradigm, the cue stimulus is presented less frequently, and participants actively maintain the task set for a longer period until the next cue is presented. Thus, in the switch trial, the effect of a cue presentation of the previous task is diminished, but the interference of the previous task set becomes greater (Sakai, 2008). The greater demand in the switch trial and the weaker effect of cue presentation of the previous task may be attributable to the greater classification accuracy of the switch trial observed in the current study.

Several previous studies have shown that switching alternative tasks was associated with the frontoparietal region in the left hemisphere (Konishi et al., 1998, 2002; Dove et al., 2000; Rushworth et al., 2002; Braver et al., 2003; Crone et al., 2006; Brass and von Cramon, 2004; Asari et al., 2005; Derrfuss et al., 2005; Yeung et al., 2006; Jimura and Braver, 2010; C. Kim et al., 2011, 2012). In the current study, when a stimulus involved perceptual uncertainty, prefrontal activity in the contralateral hemisphere became stronger, which may supplement top-down signaling to stimulus-modality-dependent occipitotemporal regions (Fig. 7), suggesting a compensatory mechanism across hemispheres. Indeed, such contralateral compensation has been reported in neuropsychological studies of patients with hemispheric stroke (Buckner et al., 1996; Cramer et al., 1997; Gold and Kertesz, 2000), which may be compatible with the complementary activation and top-down signaling under perceptual uncertainty in the current study. Interestingly, it is suggested that the right prefrontal and parietal regions are associated with stimulus-driven attention (Corbetta and Shulman, 2002), consistent with increased perceptual demand during low-coherence trials in the current study.

Previous electrophysiological studies have shown that MT activity becomes greater when subjects are perceiving random dot motion with greater coherence (Britten et al., 1992, 1993; Britten and Newsome, 1998; Mazurek et al., 2003). On the other hand, similarly to the current study, under conditions where visual stimuli involved both color and motion perceptual uncertainty, MT activity increased in high-motion coherence trials when subjects did not attend to the motion (Kayser et al., 2010); this was consistent with the previous electrophysiological studies (Britten et al., 1992, 1993; Britten and Newsome, 1998; Mazurek et al., 2003). However, when subjects did attend to motion, the direction of motion coherence modulation reversed, with MT activity becoming greater in low-motion coherence trials, suggesting top-down signaling from the frontoparietal regions (Kayser et al., 2010). The current study suggests that the top-down signal is enhanced during task switching in low-coherence trials, and that the top-down signal is associated with supplemental attention to task-relevant information when the stimulus involves multiple dimensional uncertainties (Fig. 7). Notably, consistent with a previous study (Kayser et al., 2010), in the current study, such enhanced MT activation was not observed during high-coherence trials, but rather MT activity increased in low-coherence trials $(p<0.05$ corrected within small volume in the MT region 
defined with contrast of motion vs color trials in a univariate analysis).

In conclusion, the current study elucidated one aspect of executive control. Specifically, behavioral flexibility and perceptual decision-making interact to guide goal-directed behavior, which is implemented as a distributed cortical network in the frontal and occipitotemporal regions.

\section{References}

Allport A, Styles EA, Hsieh S (1994) Shifting intentional set: exploring the dynamic control of tasks. In: Conscious and nonconscious information processing: attention and performance, VolXV. (Umilta C, Moscovitch $\mathrm{M}$ eds), pp 421-452. Cambridge, MA: Massachusetts Institute of Technology.

Aron AR, Monsell S, Sahakian BJ, Robbins TW (2004) A componential analysis of task-switching deficits associated with lesions of left and right frontal cortex. Brain 127:1561-1573.

Asari T, Konishi S, Jimura K, Miyashita Y (2005) Multiple components of lateral posterior parietal activation associated with cognitive set shifting. Neuroimage 26:694-702.

Bartels A, Zeki S (2000) The architecture of the colour centre in the human visual brain: new results and a review. Eur J Neurosci 12:172-193.

Beauchamp MS, Cox RW, Deyoe EA (1997) Graded effects of spatial and featural attention on human area MT and associated motion processing areas. J Neurophysiol 78:516-520.

Bowling JT, Friston KJ, Hopfinger JB (2020) Top-down versus bottom-up attention differentially modulate frontal-parietal connectivity. Hum Brain Mapp 41:928-942.

Braddick OJ, O'Brien JM, Wattam-Bell J, Atkinson J, Hartley T, Turner R (2001) Brain areas sensitive to coherent visual motion. Perception 30:6172 .

Brainard DH (1997) The psychophysics toolbox. Spat Vis 10:433-436.

Brass M, von Cramon DY (2004) Decomposing components of task preparation with functional magnetic resonance imaging. J Cogn Neurosci 16:609-620.

Braver TS, Reynolds JR, Donaldson DI (2003) Neural mechanisms of transient and sustained cognitive control during task switching. Neuron 39:713-726.

Britten KH, Newsome WT (1998) Tuning bandwidths for near-threshold stimuli in area MT. J Neurophysiol 80:762-770.

Britten KH, Shadlen MN, Newsome WT, Movshon JA (1992) The analysis of visual motion: a comparison of neuronal and psychophysical performance. J Neurosci 12:4745-4765.

Britten KH, Shadlen MN, Newsome WT, Movshon JA (1993) Responses of neurons in macaque MT to stochastic motion signals. Vis Neurosci 10:1157-1169.

Buckner RL, Corbetta M, Schatz J, Raichle ME, Petersen SE (1996) Preserved speech abilities and compensation following prefrontal damage. Proc Natl Acad Sci USA 93:1249-1253.

Camilleri JA, Müller VI, Fox P, Laird AR, Hoffstaedter F, Kalenscher T, Eickhoff SB (2018) Definition and characterization of an extended multiple-demand network. Neuroimage 165:138-147.

Casey BJ, Trainor RJ, Orendi JL, Schubert AB, Nystrom LE, Giedd JN, Castellanos FX, Haxby JV, Noll DC, Cohen JD, Forman SD, Dahl RE, Rapoport JL (1997) A developmental functional MRI study of prefrontal activation during performance of a Go-No-Go task. J Cogn Neurosci 9:835-847.

Chen MY, Jimura K, White CN, Maddox WT, Poldrack RA (2015) Multiple brain networks contribute to the acquisition of bias in perceptual decision-making. Front Neurosci 9:63.

Cole MW, Reynolds JR, Power JD, Repovs G, Anticevic A, Braver TS (2013) Multi-task connectivity reveals flexible hubs for adaptive task control. Nat Neurosci 16:1348-1355.

Corbetta M, Miezin FM, Dobmeyer S, Shulman GL, Petersen SE (1991) Selective and divided attention during visual discriminations of shape, color, and speed: functional-anatomy by positron emission tomography. J Neurosci 11:2383-2402.

Corbetta M, Shulman GL (2002) Control of goal-directed and stimulusdriven attention in the brain. Nat Rev Neurosci 3:201-215.
Cramer SC, Nelles G, Benson RR, Kaplan JD, Parker RA, Kwong KK, Kennedy DN, Finklestein SP, Rosen BR (1997) A functional MRI study of subjects recovered from hemiparetic stroke. Stroke 28:2518-2527.

Crone EA, Wendelken C, Donohue SE, Bunge SA (2006) Neural evidence for dissociable components of task-switching. Cereb Cortex 16:475-486.

Derrfuss J, Brass M, Neumann J, von Cramon DY (2005) Involvement of the inferior frontal junction in cognitive control: meta-analyses of switching and Stroop studies. Hum Brain Mapp 25:22-34.

Desimone R, Duncan J (1995) Neural mechanisms of selective visual-attention. Annu Rev Neurosci 18:193-222.

Dosenbach NUF, Visscher KM, Palmer ED, Miezin FM, Wenger KK, Kang HSC, Burgund ED, Grimes AL, Schlaggar BL, Petersen SE (2006) A core system for the implementation of task sets. Neuron 50:799-812.

Dove A, Pollmann S, Schubert T, Wiggins CJ, von Cramon DY (2000) Prefrontal cortex activation in task switching: an event-related fMRI study. Brain Res Cogn Brain Res 9:103-109.

Duncan J (2010) The multiple-demand (MD) system of the primate brain: mental programs for intelligent behaviour. Trends Cogn Sci 14:172-179.

Egner T, Hirsch J (2005) Cognitive control mechanisms resolve conflict through cortical amplification of task-relevant information. Nat Neurosci 8:1784-1790.

Eklund A, Nichols TE, Knutsson H (2016) Cluster failure: inflated false positives for fMRI. Proc Natl Acad Sci USA 113:7900-7905.

Erwin RJ, Gur RC, Gur RE, Skolnick B, Mawhinney-Hee M, Smailis J (1992) Facial emotion discrimination: I. Task construction and behavioral findings in normal subjects. Psychiatry Res 42:231-240.

Friston KJ, Harrison L, Penny W (2003) Dynamic causal modelling. Neuroimage 19:1273-1302.

Friston KJ, Litvak V, Oswal A, Razi A, Stephan KE, Wijk BC, Ziegler G, Zeidman P (2016) Bayesian model reduction and empirical Bayes for group (DCM) studies. Neuroimage 128:413-431.

Fuster JM (2015) The prefrontal Cortex, Ed 5. London: Academic Press.

Glasser MF, Coalson TS, Robinson EC, Hacker CD, Harwell J, Yacoub E, Ugurbil K, Andersson J, Beckmann CF, Jenkinson M, Smith SM, van Essen DC (2016) A multi-modal parcellation of human cerebral cortex. Nature 536:171-178.

Gold BT, Kertesz A (2000) Right hemisphere semantic processing of visual words in an aphasic patient: an fMRI study. Brain Lang 73:456-465.

Gold JI, Shadlen MN (2007) The neural basis of decision making. Annu Rev Neurosci 30:535-574.

He Q, Huang X, Zhang S, Turel O, Ma L, Bechara A (2019) Dynamic causal modeling of insular, striatal, and prefrontal cortex activities during a food-specific Go/NoGo task. Biol Psychiatry Cogn Neurosci Neuroimaging 4:1080-1089.

Huk AC, Dougherty RF, Heeger DJ (2002) Retinotopy and functional subdivision of human areas MT and MST. J Neurosci 22:7195-7205.

Jersild AT (1927) Mental set and shift. Arch Psychol 89:5-82.

Jiang J, Wagner AD, Egner T (2018) Integrated externally and internally generated task predictions jointly guide cognitive control in prefrontal cortex. eLife 16:e39497.

Jimura K, Braver TS (2010) Age-related shifts in brain activity dynamics during task switching. Cereb Cortex 20:1420-1431.

Jimura K, Konishi S, Miyashita Y (2004) Dissociable concurrent activity of lateral and medial frontal lobe during negative feedback processing. Neuroimage 22:1578-1586.

Jimura K, Cazalis F, Stover ER, Poldrack RA (2014) The neural basis of task switching changes with skill acquisition. Front Hum Neurosci 8:339.

Kastner S, Ungerleider LG (2000) Mechanisms of visual attention in the human cortex. Annu Rev Neurosci 23:315-341.

Kayser AS, Erickson DT, Buchsbaum BR, D’Esposito M (2010) Neural representations of relevant and irrelevant features in perceptual decision making. J Neurosci 30:15778-15789.

Kim C, Johnson NF, Cilles SE, Gold BT (2011) Common and distinct mechanisms of cognitive flexibility in prefrontal cortex. J Neurosci 31:47714779.

Kim C, Cilles SE, Johnson NF, Gold BT (2012) Domain general and domain preferential brain regions associated with different types of task switching: a meta-analysis. Hum Brain Mapp 33:130-142.

Kim JN, Shadlen MN (1999) Neural correlates of a decision in the dorsolateral prefrontal cortex of the macaque. Nat Neurosci 2:176-185. 
Konishi S, Nakajima K, Uchida I, Kameyama M, Nakahara K, Sekihara K, Miyashita Y (1998) Transient activation of inferior prefrontal cortex during cognitive set shifting. Nat Neurosci 1:80-84.

Konishi S, Hayashi T, Uchida I, Kikyo H, Takahashi E, Miyashita Y (2002) Hemispheric asymmetry in human lateral prefrontal cortex during cognitive set shifting. Proc Natl Acad Sci USA 99:7803-7808.

Kriegeskorte N, Goebel R, Bandettini P (2006) Information-based functional brain mapping. Proc Natl Acad Sci USA 103:3863-3868.

Kriegeskorte N, Simmons WK, Bellgowan PS, Baker CI (2009) Circular analysis in systems neuroscience: the dangers of double dipping. Nat Neurosci 12:535-540.

Krystal JH, Karper LP, Seibyl JP, Freeman GK, Delaney R, Bremner JD, Heninger GR, Bowers MB, Charney DS (1994) Subanesthetic effects of the noncompetitive NMDA antagonist, ketamine, in humans: psychotomimetic, perceptual, cognitive, and neuroendocrine responses. Arch Gen Psychiatry 51:199-214.

Kumano H, Suda Y, Uka T (2016) Context-dependent accumulation of sensory evidence in the parietal cortex underlies flexible task switching. J Neurosci 36:12192-12202.

Loose LS, Wisniewski D, Rusconi M, Goschke T, Haynes JD (2017) Switchindependent task representations in frontal and parietal cortex. J Neurosci 37:8033-8042.

MacDonald AW, Cohen JD, Stenger VA, Carter CS (2000) Dissociating the role of the dorsolateral prefrontal and anterior cingulate cortex in cognitive control. Science 288:1835-1838.

Mante V, Sussillo D, Shenoy KV, Newsome WT (2013) Context-dependent computation by recurrent dynamics in prefrontal cortex. Nature 503:7884.

Mazurek ME, Roitman JD, Ditterich J, Shadlen MN (2003) A role for neural integrators in perceptual decision making. Cereb Cortex 13:1257-1269.

Miller EK, Cohen JD (2001) An integrative theory of prefrontal cortex function. Annu Rev Neurosci 24:167-202.

Miyake A, Friedman NP, Emerson MJ, Witzki AH, Howerter A, Wager TD (2000) The unity and diversity of executive functions and their contributions to complex "frontal lobe" tasks: a latent variable analysis. Cogn Psychol 41:49-100.

Moeller S, Yacoub E, Olman CA, Auerbach E, Strupp J, Harel N, Uğurbil K (2010) Multiband multislice GE-EPI at 7 tesla, with 16-fold acceleration using partial parallel imaging with application to high spatial and temporal whole-brain fMRI. Magn Reson Med 63:1144-1153.

Monsell S (2003) Task switching. Trends Cogn Sci 7:134-140.

Motter BC (1994) Neural correlates of attentive selection for color or luminance in extrastriate area V4. J Neurosci 14:2178-2189.

Nee DE, D'Esposito M (2016) The hierarchical organization of the lateral prefrontal cortex. eLife 5:e12112.

Newsome WT, Pare EB (1988) A selective impairment of motion perception following lesions of the middle temporal visual area (MT). J Neurosci 8:2201-2211.

Osada T, Adachi Y, Miyamoto K, Jimura K, Setsuie R, Miyashita Y (2015) Dynamically allocated hub in task-evoked network predicts the vulnerable prefrontal locus for contextual memory retrieval in macaques. PLoS Biol 13:e1002177.

Palmer J, Huk AC, Shadlen MN (2005) The effect of stimulus strength on the speed and accuracy of a perceptual decision. J Vis 5:376-404.

Pelli DG (1997) The VideoToolbox software for visual psychophysics: transforming numbers into movies. Spat Vis 10:437-442.

Penny WD, Stephan KE, Mechelli A, Friston KJ (2004) Comparing dynamic causal models. Neuroimage 22:1157-1172.
Penny WD, Stephan KE, Daunizeau J, Rosa MJ, Friston KJ, Schofield TM, Leff AP (2010) Comparing families of dynamic causal models. PLoS Comput Biol 6:e1000709.

Purcell BA, Kiani R (2016) Hierarchical decision processes that operate over distinct timescales underlie choice and changes in strategy. Proc Natl Acad Sci USA 113:E4531-E4540.

Qiao L, Zhang L, Chen A, Egner T (2017) Dynamic trial-by-trial recoding of task-set representations in the frontoparietal cortex mediates behavioral flexibility. J Neurosci 37:11037-11050.

Roberts AC, Robbins TW, Weiskrantz L (1998) The prefrontal cortex: executive and cognitive functions. Oxford:Oxford University Press.

Rogers RD, Monsell S (1995) Costs of a predictable switch between simple cognitive tasks. J Exp Psychol Gen 124:207-231.

Romo R, Hernandez A, Zainos A, Salinas E (1998) Somatosensory discrimination based on cortical microstimulation. Nature 392:387-390.

Rushworth MF, Passingham RE, Nobre AC (2002) Components of switching intentional set. J Cogn Neurosci 14:1139-1150.

Sakai K (2008) Task set and prefrontal cortex. Annu Rev Neurosci 31:129245.

Sarafyazd M, Jazayeri M (2019) Hierarchical reasoning by neural circuits in the frontal cortex. Science 364:eaav8911.

Schein SJ, Desimone R (1990) Spectral properties of V4 neurons in the macaque. J Neurosci 10:3369-3389.

Shackman AJ, Salomons TV, Slagter HA, Fox AS, Winter JJ, Davidson RJ (2011) The integration of negative affect, pain and cognitive control in the cingulate cortex. Nat Rev Neurosci 12:154-167.

Seeley WW, Menon V, Schatzberg AF, Keller J, Glover GH, Kenna H, Reiss AL, Greicius MD (2007) Dissociable intrinsic connectivity networks for salience processing and executive control. J Neurosci 27:2349-2356.

Shadlen MN, Newsome WT (1994) Noise, neural codes and cortical organization. Curr Opin Neurobiol 4:569-579.

Shadlen MN, Britten KH, Newsome WT, Movshon JA (1996) A computational analysis of the relationship between neuronal and behavioral responses to visual motion. J Neurosci 16:1486-1510.

Stephan KE, Penny WD, Moran RJ, den Ouden HE, Daunizeau J, Friston KJ (2010) Ten simple rules for dynamic causal modeling. Neuroimage 49:3099-3109.

Tomita H, Ohbayashi M, Nakahara K, Hasegawa I, Miyashita Y (1999) Topdown signal from prefrontal cortex in executive control of memory retrieval. Nature 401:699- 703

Treisman AM, Kanwisher NG (1998) Perceiving visually presented objects: recognition, awareness, and modularity. Curr Opin Neurobiol 8:218-226.

Waskom ML, Kumaran D, Gordon AM, Rissman J, Wagner AD (2014) Frontoparietal representations of task context support the flexible control of goal-directed cognition. J Neurosci 34:10743-10755.

Worsley DB, Friston KJ (1995) Analysis of fMRI time-series revisited again. Neuroimage 2:173-181.

Yarkoni T, Poldrack RA, Nichols TE, van Essen DC, Wager TD (2011) Large-scale automated synthesis of human functional neuroimaging data. Nat Methods 8:665-670.

Yeung N, Nystrom LE, Aronson JA, Cohen JD (2006) Between-task competition and cognitive control in task switching. J Neurosci 26:1429-1438.

Zeki S (1973) Colour coding in rhesus monkey prestriate cortex. Brain Res 53:422-427.

Zeki S, Watson JD, Lueck CJ, Friston KJ, Kennard C, Frackowiak RS (1991) A direct demonstration of functional specialization in human visual cortex. J Neurosci 11:641-649.

Zhang J, Kriegeskorte N, Carlin JD, Rowe JB (2013) Choosing the rules: distinct and overlapping frontoparietal representations of task rules for perceptual decisions. J Neurosci 33:11852-11862. 